\title{
Bond Strength of Universal Adhesives to Dentin: A Systematic Review and Meta-Analysis
}

\author{
Louis Hardan ${ }^{1}\left(\mathbb{D}\right.$, Rim Bourgi ${ }^{1}\left(\mathbb{D}\right.$, Naji Kharouf ${ }^{2,3, *}$, Davide Mancino ${ }^{2,3}$, Maciej Zarow ${ }^{4}$, Natalia Jakubowicz ${ }^{4}$, \\ Youssef Haikel ${ }^{2}$ and Carlos Enrique Cuevas-Suárez ${ }^{5, *}$ (i)
}

1 Department of Restorative Dentistry, School of Dentistry, Saint-Joseph University, 11072180 Beirut, Lebanon; louis.hardan@usj.edu.lb (L.H.); rim.bourgi@net.usj.edu.lb (R.B.)

2 Department of Biomaterials and Bioengineering, INSERM UMR_S 1121, Biomaterials and Bioengineering, 67000 Strasbourg, France; endodontiefrancaise@outlook.com (D.M.); youssef.haikel@unistra.fr (Y.H.)

3 Department of Endodontics, Faculty of Dental Medicine, Strasbourg University, 67000 Strasbourg, France

4 Private Practice, “NZOZ SPS Dentist” Dental Clinic and Postgraduate Course Centre, pl. Inwalidow 7/5, 30-033 Cracow, Poland; dentist@dentist.com.pl (M.Z.); nljakubowicz@gmail.com (N.J.)

5 Dental Materials Laboratory, Academic Area of Dentistry, Autonomous University of Hidalgo State, Circuito Ex Hacienda La Concepción S/N, San Agustín Tlaxiaca, Hidalgo 42160, Mexico

* Correspondence: dentistenajikharouf@gmail.com (N.K.); cecuevas@uaeh.edu.mx (C.E.C.-S.); Tel.: +33-36-6752-2841 (N.K.); +52-177172000 (C.E.C.-S.)

check for

updates

Citation: Hardan, L.; Bourgi, R.; Kharouf, N.; Mancino, D.; Zarow, M.; Jakubowicz, N.; Haikel, Y.;

Cuevas-Suárez, C.E. Bond Strength of Universal Adhesives to Dentin: A Systematic Review and Meta-Analysis. Polymers 2021, 13, 814. https://doi.org/10.3390/ polym13050814

Academic Editor: Andrea Scribante

Received: 14 February 2021

Accepted: 4 March 2021

Published: 7 March 2021

Publisher's Note: MDPI stays neutral with regard to jurisdictional claims in published maps and institutional affiliations.

Copyright: (C) 2021 by the authors Licensee MDPI, Basel, Switzerland This article is an open access article distributed under the terms and conditions of the Creative Commons Attribution (CC BY) license (https:// creativecommons.org/licenses/by/ $4.0 /)$.

\begin{abstract}
Currently, the availability of a wide variety of universal adhesives makes it difficult for clinicians to choose the correct system for specific bonding situations to dentin substrate. This study aimed to determine whether there are any alternative techniques or additional strategies available to enhance the bond strength of universal adhesives to dentin through a systematic review and meta-analysis. Two reviewers executed a literature search up to September 2020 in four electronic databases: PubMed, ISI Web of Science, Scopus, and EMBASE. Only in vitro studies that reported the dentin bond strength of universal adhesives using additional strategies were included. An analysis was carried out using Review Manager Software version 5.3.5 (The Nordic Cochrane Centre, The Cochrane Collaboration, Copenhagen, Denmark). The methodological quality of each in vitro study was assessed according to the parameters of a previous systematic. A total of 5671 potentially relevant studies were identified. After title and abstract examination, 74 studies remained in systematic review. From these, a total of 61 studies were included in the meta-analysis. The bond strength of universal adhesives to dentin was improved by the use of one of the following techniques: Previous application of matrix metalloproteinases (MMP) inhibitors $(p<0.001)$, prolonged application time $(p=0.007)$, scrubbing technique $(p<0.001)$, selective dentin etching $(p<0.001)$, nonatmospheric plasma $(p=0.01)$, ethanol-wet bonding $(p<0.01)$, prolonged blowing time $(p=0.02)$, multiple layer application $(p=0.005)$, prolonged curing time $(p=0.006)$, and hydrophobic layer coating $(p<0.001)$. On the other hand, the use of a shortened application time $(p=0.006)$, and dentin desensitizers $(p=0.01)$ impaired the bond strength of universal adhesives to dentin. Most of the analyses performed showed a high heterogenicity. The in vitro evidence suggests that the application of universal adhesives using some alternative techniques or additional strategies may be beneficial for improving their bonding performance to dentin. This research received no external funding. Considering that this systematic review was carried out only with in vitro studies, registration was not performed.
\end{abstract}

Keywords: adhesion; dental adhesive; dental bonding; dentin bonding agent; universal adhesive

\section{Introduction}

Despite the enormous improvements made in adhesive technology throughout the last 50 years, there are still some unresolved problems regarding the durability of the adhesive interface [1]. Impervious seal between resin and dentin substrate remains difficult 
to achieve [2], and it is doubtful that an ideal interdiffusion of the adhesive system inside collagen scaffold can be produced in a harmonious fashion [3]. In fact, hydrolytic degradation combined by the action of dentinal matrix enzymes are thought to destabilize the tooth-adhesive bond and break down the unprotected collagen fibrils [4]. Even though sufficient resin-dentin bonding is generally immediately reached, lessened bonding efficiency arises with time [5].

All in all, the actual mechanisms of degradation are far from being fully understood. In this sense, it is necessary to strictly characterize the recommendations of each company and to follow them; otherwise, deterioration of the adhesive could occur [6]. Achieving adequate hybridization within collagen fibrils and the stability of resin-dentin interface are of key importance [7]. It is fundamental to recognize that the mechanism of hybridization in which an interdiffusion zone, also called: "Hybrid layer", is created, fulfills the occurrence of the micromechanical retention of the restoration [8].

The quality of adhesion relies on numerous factors and may vary according to the adhesive system used which can be either the self-etch or the etch-and-rinse [9]. A simplification of the classical concept of the dental bonding by means of a less-sensitive technique, faster application, and various optional applicability is possible nowadays with the introduction of universal adhesives [10]. Based on this, universal adhesives constitute one type of all-in-one adhesives with the presence of both hydrophobic and hydrophilic components on the same bottle [11], and according to manufacturers, they can be used in both direct and indirect substrates [12]. However, the stability and durability of dentin-adhesive interface generated by these new multimode adhesive systems continue to be questionable [13]. One of the major concerns of these systems was related to the increase of nanoleakage after aging, and thus leading to a limited bond durability [14]. The issue is that until now, there has been no golden pattern protocol for achieving stable and optimal adhesion of universal adhesive systems to dentin.

Furthermore, improvement in bond strength can be realized by means of numerous strategies, and therefore, suggested by many authors $[15,16]$. Thus, the aim of this study was to evaluate whether, in vitro, the immediate and long-term bonding performance of universal adhesives to dentin would be improved by the use of any additional strategies through a systematic review and meta-analysis. The null hypothesis of the present study was that there would be no difference in immediate and long-term bond strength to dentin substrate when using universal adhesives with any alternative technique other than the instructions of the manufacturer.

\section{Materials and Methods}

This systematic review and meta-analysis was performed in accordance with the PRISMA 2020 statement [17]. The following PICOS framework was used: Population, permanent dentin substrate (human); intervention, application of universal adhesives with additional steps or different techniques; control, application of universal adhesives according to manufacturer instructions; outcome, bond strength; and study design, in vitro studies. The research question was: "Is there any technique or additional step available to improve the bond strength of universal adhesives to dentin"?

\subsection{Literature Search}

The literature search was conducted by two independent reviewers (C.E.C.-S. and R.B.) up to 26 September 2020. The following four electronic databases were screened to identify manuscripts that could be included: PubMed (MedLine), ISI Web of Science, Scopus, and Embase. The inter-examiner agreement was quantified using the kappa coefficient. The keywords and search strategy used in PubMed are listed in Table 1. The full search strategy for ISI Web of Science, Scopus, and EMBASE databases is presented as supplementary material (Tables S1-S3). The reviewers also performed a hand search of reference lists of included articles for identification of additional papers. Following the initial screening, 
all studies were imported into Mendeley Desktop 1.17.11 software (Glyph \& Cog, LLC, London, UK) to eliminate duplicates.

Table 1. Search strategy used in PubMed.

\begin{tabular}{|c|c|}
\hline \multicolumn{2}{|r|}{ Search Strategy } \\
\hline$\# 1$ & $\begin{array}{c}\text { Universal adhesives OR Universal adhesive OR Universal simplified adhesive } \\
\text { systems OR Universal Dental Adhesives OR Multipurpose adhesives OR } \\
\text { multi-purpose adhesives OR multimode adhesives OR multi-mode adhesives } \\
\text { or universal bonding agent }\end{array}$ \\
\hline$\# 2$ & $\begin{array}{l}\text { Bond OR Bonding OR Dental bonding OR Bonding efficacy OR bond strength } \\
\text { OR Bonding performance OR bonding effectiveness OR Bond performance OR } \\
\text { adhesive properties OR microtensile strength OR Micro-tensile strength OR } \\
\text { bonding properties OR Microtensile bond strength OR shear bond strength OR } \\
\text { microshear bond strength OR performance }\end{array}$ \\
\hline \# 3 & Dentine OR Dentin \\
\hline$\# 4$ & $\# 1$ and $\# 2$ and \#3 \\
\hline
\end{tabular}

\subsection{Study Selection}

Two reviewers (R.B. and C.E.C.-S.) individually assessed the titles and abstracts of all studies. Manuscripts for full-text reviews were selected according to the following eligibility criteria: (1) In vitro studies reporting the effect of the use of additional steps for the application of universal adhesive systems in the bond strength to dentin; (2) evaluating the bond strength of universal adhesive systems to dentin with a resin-based material as an antagonist; (3) including a control group in which universal adhesive was applied according to manufacturers' instructions; (4) including mean and standard deviation (SD) data in MPa on shear, micro-shear, tensile, and micro-tensile bond tests. Only manuscripts published in the English language were considered. Manuscripts that involved different substrates further than those recognized in the inclusion criteria were not considered. Case reports, pilot studies, case series, and reviews were also excluded. Full copies of all of the potentially relevant studies were assessed. Papers that seemed to meet the inclusion criteria or had insufficient data in the title and abstract to produce a clear decision were designated for full analysis. The full-text manuscripts were considered independently in duplicate by two review authors. Any discrepancy concerning the eligibility of the included studies was decided and resolved through discussion and agreement by a third reviewer (L.H.). Only papers that satisfied the eligibility criteria listed were included.

\subsection{Data Extraction}

Data of concern from the involved studies were extracted using Microsoft Office Excel 2019 (Microsoft Corporation, Redmond, WA, USA). These data comprised the year of publication, type of tooth storage, strategy evaluated for bonding to dentin, type of bond strength test evaluated, and adhesive system used. If any information was missing, the corresponding authors of the included studies were notified twice via an e-mail to retrieve the missing data. If the authors did not respond within 2 weeks of the first contact, the missing information was not included.

\subsection{Quality Assessment}

The methodological quality of each in vitro study was assessed by two reviewers (L.H. and R.B.), according to the parameters of a previous systematic review [16]. The risk of bias in each article was evaluated according to the description of the following parameters: Specimen randomization; single-operator protocol implementation; blinding of the testing machine operator; the presence of a control group; standardization of the sample preparation; failure mode evaluation; use of all materials according to the manufacturer's instructions; and description of the sample size calculation. If the authors reported the 
parameter, the study received a "YES" for that specific parameter. In the case of missing information, the parameter received a "NO." The risk of bias was classified according to the sum of "YES" answers received: 1 to 3 indicated a high bias, 4 to 6 medium, and 7 to 8 indicated a low risk of bias.

\subsection{Statistical Analysis}

Meta-analysis was performed using Review Manager Software version 5.3.5 (The Nordic Cochrane Centre, The Cochrane Collaboration, Copenhagen, Denmark). The analysis was carried out using the random-effects model and pooled-effect estimates were obtained by comparing the standardized mean difference between bond strength values obtained from the control and experimental. In addition, immediate and long-term bond strength data were analyzed separately. In studies where several experimental groups were compared with the same control group, data from the experimental groups (mean, standard deviation, and sample size) were combined for the meta-analysis [18]. A $p$-value $<0.05$ was considered statistically significant. Statistical heterogeneity of the treatment effect among studies was assessed using the Cochran $\mathrm{Q}$ test and the inconsistency $\mathrm{I}^{2}$ test.

\section{Results}

A total of 5671 publications were retrieved in all databases. A flowchart that summarizes the study selection process according to the PRISMA Statement is shown in Figure 1. The literature review retrieved 4303 manuscripts for the initial examination after the duplicates were removed. Of these, 4198 studies were excluded after reviewing the titles and abstracts. In total, 105 studies were examined by full-text reading. Of these, 31 studies were excluded [19-47], the reasons for exclusion are mentioned in the supplementary material (Table S4), totalizing 74 articles in the qualitative analysis. Of these, 13 studies were excluded: In 9 studies, there were not enough study groups to perform any comparison [39,40,48-54], 2 studies did not have any control group [38,55], 1 study combined data from different generations of adhesives [56], and 1 study did not present the mean and SD [57]. Thus, 61 studies were included in the meta-analysis. The inter-examiner agreement was excellent (kappa coefficient $=0.87$ ).

The qualitative synthesis of the studies included in this systematic review are summarized in Table 2. Several strategies were identified: Air abrasion [58,59], application of crosslinking agents [57,60-65], application of dentin desensitizers [66,67], use of matrix metalloproteinases (MMP) inhibitors [10,68-77], the use of the ethanol-wet bonding technique [78], application of an extra hydrophobic resin layer [56,79-85], dentin pretreatment with laser [55,86-92], multiple layer application [93-99], non-atmospheric plasma [100,101], prolonged curing time [102,103], prolonged blowing time [104-106], application of the adhesive using a scrubbing technique [107-109], selective dentin etching [110-112], prolonged application time [104,105,113], shortened application time [99,106,114-116], application of the adhesive using an electric-current [48], combining of phosphoric acid with chlorhexidine [39], using ozone as cavity disinfectant [50], pre-warming of adhesive bottles [52], air-blowing with warm-air [53] smear layer deproteinization [54], and removal of the oxygen inhibited layer [40]. 
PRISMA FLOW DIAGRAM

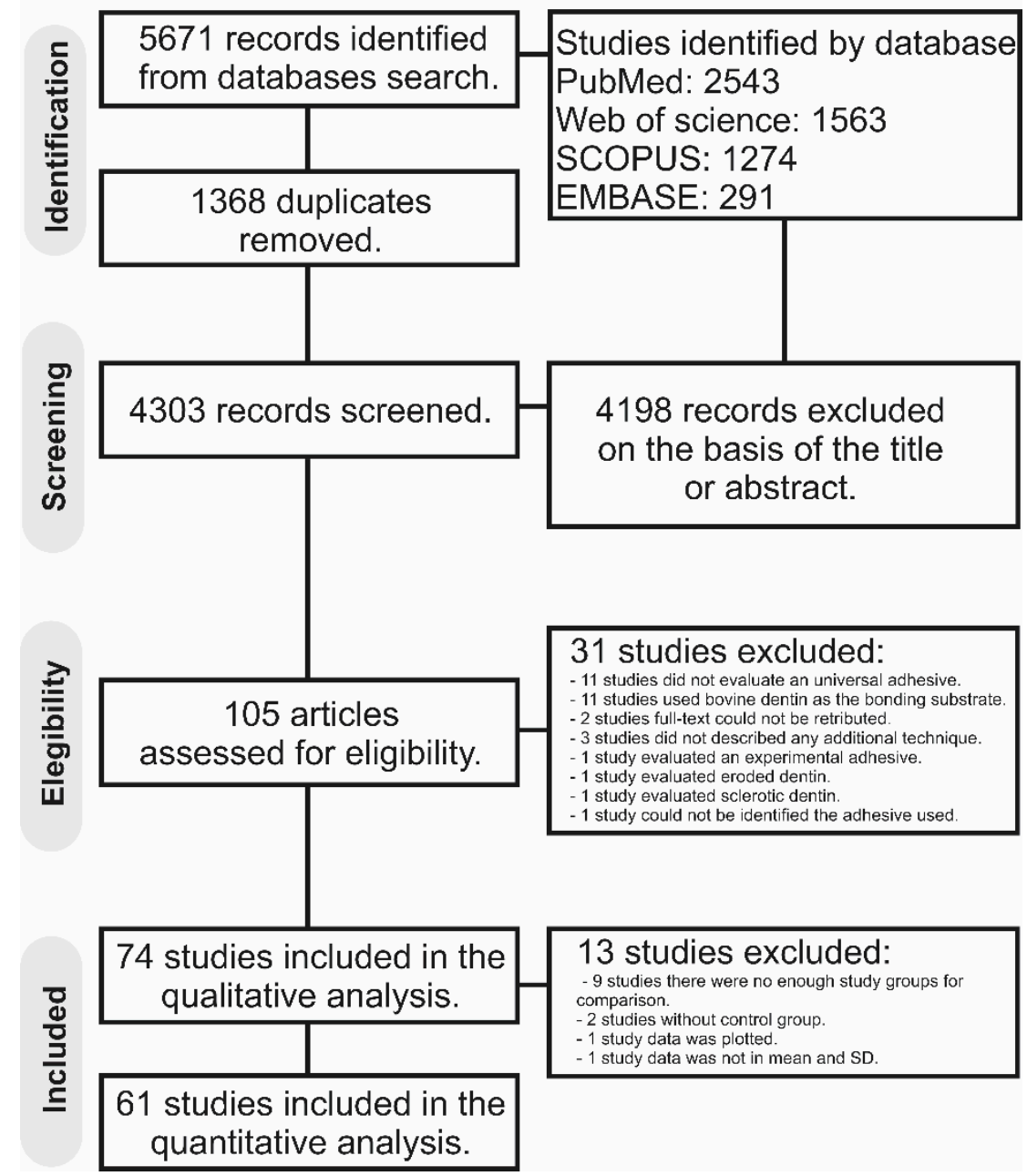

Figure 1. Prisma flow diagram of the study.

Table 2. Demographic and study design data of the included studies.

\begin{tabular}{|c|c|c|c|c|}
\hline Study & Aging/Storage & Strategy & Bond Strength Test & Adhesive System Used \\
\hline Flury, 2017 & $24 \mathrm{~h}$ of water storage at $37^{\circ} \mathrm{C}$ & Air Abrasion & Dentin $\mu$ SBS & $\begin{array}{l}\text { Scotchbond Universal } \\
\text { (3M ESPE) }\end{array}$ \\
\hline Sutil, 2017 & $24 \mathrm{~h}$ of water storage at $37^{\circ} \mathrm{C}$ & Air Abrasion & Dentin $\mu \mathrm{TBS}$ & $\begin{array}{l}\text { Scotchbond Universal } \\
\text { (3M ESPE) }\end{array}$ \\
\hline Bacelar-Sá, 2017 & $\begin{array}{c}24 \mathrm{~h} \text { of water storage at } 37^{\circ} \mathrm{C} \\
6 \text { months of water storage at } \\
37^{\circ} \mathrm{C}\end{array}$ & $\begin{array}{l}\text { Application of collagen } \\
\text { crosslinking agents }\end{array}$ & Dentin $\mu \mathrm{TBS}$ & $\begin{array}{c}\text { Scotchbond Universal } \\
\text { (3M ESPE) } \\
\text { Prime \& Bond Elect } \\
\text { (Dentsply) } \\
\text { All-Bond } 3 \text { (Bisco Inc.) } \\
\text { G-Aenial (GC Corp.) }\end{array}$ \\
\hline Baena, 2020 & $\begin{array}{c}24 \mathrm{~h} \text { of water storage at } 37^{\circ} \mathrm{C} \\
10,000 \text { thermocycles between } 5 \\
\text { and } 55^{\circ} \mathrm{C}\end{array}$ & $\begin{array}{l}\text { Application of collagen } \\
\text { crosslinking agents }\end{array}$ & Dentin $\mu$ TBS & $\begin{array}{c}\text { OptiBond FL (Kerr) } \\
\text { Scotchbond Universal } \\
\text { (3M ESPE) }\end{array}$ \\
\hline Cha, 2016 & $24 \mathrm{~h}$ of water storage at $37^{\circ} \mathrm{C}$ & $\begin{array}{l}\text { Application of collagen } \\
\text { crosslinking agents }\end{array}$ & Dentin SBS & $\begin{array}{l}\text { Scotchbond Universal } \\
\text { (3M ESPE) }\end{array}$ \\
\hline de Lima, 2018 & $24 \mathrm{~h}$ of water storage at $37^{\circ} \mathrm{C}$ & $\begin{array}{l}\text { Application of collagen } \\
\text { crosslinking agents }\end{array}$ & Dentin $\mu$ TBS & $\begin{array}{l}\text { Prime \& Bond Elect } \\
\text { (Dentsply) }\end{array}$ \\
\hline
\end{tabular}


Table 2. Cont.

\begin{tabular}{|c|c|c|c|c|}
\hline Study & Aging/Storage & Strategy & Bond Strength Test & Adhesive System Used \\
\hline Kaynar, 2020 & $\begin{array}{l}10,000 \text { thermocycles } \\
\text { between } 5 \text { and } 55^{\circ} \mathrm{C}\end{array}$ & $\begin{array}{l}\text { Application of collagen } \\
\text { crosslinking agents }\end{array}$ & Dentin $\mu$ TBS & $\begin{array}{l}\text { Peak Universal Bond } \\
\text { (Ultradent Products Inc.) } \\
\text { G-Premio Bond (GC Corp) }\end{array}$ \\
\hline Kusdemir, 2015 & $48 \mathrm{~h}$ of water storage at $37^{\circ} \mathrm{C}$ & $\begin{array}{l}\text { Application of collagen } \\
\text { crosslinking agents }\end{array}$ & Dentin $\mu$ TBS & $\begin{array}{c}\text { Single Bond Universal } \\
\text { (3M ESPE) }\end{array}$ \\
\hline Paulose, 2017 & $\begin{array}{l}24 \mathrm{~h} \text { of water storage at } 37^{\circ} \mathrm{C} \\
1 \text { year of water storage at } 37^{\circ} \mathrm{C}\end{array}$ & $\begin{array}{l}\text { Application of collagen } \\
\text { crosslinking agents }\end{array}$ & Dentin $\mu$ TBS & $\begin{array}{c}\text { Single Bond Universal } \\
\text { (3M ESPE) } \\
\text { Adper Scotchbond } \\
\text { Multi-Purpose Plus } \\
\text { (3M ESPE) }\end{array}$ \\
\hline Zhang, 2020 & $\begin{array}{l}24 \mathrm{~h} \text { of water storage at } 37^{\circ} \mathrm{C} \\
1 \text {-month collagenase ageing }\end{array}$ & $\begin{array}{l}\text { Application of collagen } \\
\text { crosslinking agents }\end{array}$ & Dentin $\mu$ TBS & $\begin{array}{c}\text { Single Bond Universal } \\
\text { (3M ESPE) }\end{array}$ \\
\hline Luong, 2020 & $1 \mathrm{~h}$ of water storage at $37^{\circ} \mathrm{C}$ & $\begin{array}{l}\text { Application of dentin } \\
\text { desensitizer }\end{array}$ & Dentin $\mu$ SBS & $\begin{array}{c}\text { Parkell Universal } \\
\text { Adhesive PBOND (Parkell, } \\
\text { Edgewood, NY, USA) } \\
\text { Clearfil SE } 2 \text { (Kuraray } \\
\text { Noritake) }\end{array}$ \\
\hline iso, 2016 & $\begin{array}{l}1000 \text { thermal cycles between } \\
5^{\circ} \mathrm{C} \text { and } 55^{\circ} \mathrm{C}\end{array}$ & $\begin{array}{l}\text { Application of dentin } \\
\text { desensitizer }\end{array}$ & Dentin $\mu$ TBS & $\begin{array}{l}\text { Clearfil Universal Bond } \\
\text { (Kuraray Noritake) }\end{array}$ \\
\hline Bravo, 2017 & $\begin{array}{l}(72 \mathrm{~h}, 3 \text { months, } \\
6 \text { months) of storage in } \\
\text { distilled water at room } \\
\text { temperature }\end{array}$ & $\begin{array}{c}\text { Application of matrix } \\
\text { metalloproteinases (MMP) } \\
\text { inhibitors }\end{array}$ & Dentin $\mu$ TBS & $\begin{array}{c}\text { Adper Scotchbond 1XT } \\
\text { (3M ESPE) } \\
\text { Adper Prompt L-Pop } \\
\text { (3M ESPE) } \\
\text { Single Bond Universal } \\
\text { (3M ESPE) }\end{array}$ \\
\hline Bravo, 2017 (b) & $\begin{array}{l}(72 \mathrm{~h}, 3 \text { months, } \\
6 \text { months) of storage in } \\
\text { distilled water at room } \\
\text { temperature }\end{array}$ & $\begin{array}{l}\text { Application of MMP } \\
\text { inhibitors }\end{array}$ & Dentin SBS & $\begin{array}{c}\text { Adper Scotchbond 1XT } \\
\text { (3M ESPE) } \\
\text { Adper Prompt L-Pop } \\
\text { (3M ESPE) } \\
\text { Single Bond Universal } \\
\text { (3M ESPE) }\end{array}$ \\
\hline Chaharom, 2018 & $\begin{array}{l}500 \text { thermal cycles between } \\
\qquad 5^{\circ} \mathrm{C} \text { and } 55^{\circ} \mathrm{C}\end{array}$ & $\begin{array}{l}\text { Application of MMP } \\
\text { inhibitors }\end{array}$ & Dentin $\mu$ TBS & $\begin{array}{c}\text { All-Bond } 3 \text { (Bisco Inc.) } \\
\text { Clearfil SE Bond (Kuraray } \\
\text { Noritake) } \\
\text { All Bond Universal } \\
\text { (Bisco Inc.) }\end{array}$ \\
\hline Giacomini, 2020 & $\begin{array}{c}24 \mathrm{~h} \text { of storage in artificial } \\
\text { saliva at } 37^{\circ} \mathrm{C} \\
6 \text { months of storage in artificial } \\
\text { saliva at } 37^{\circ} \mathrm{C}\end{array}$ & $\begin{array}{l}\text { Application of MMP } \\
\text { inhibitors }\end{array}$ & Dentin $\mu$ TBS & $\begin{array}{c}\text { Single Bond Universal } \\
\text { (3M ESPE) } \\
\text { Adper Single Bond } 2 \\
\text { (3M ESPE) }\end{array}$ \\
\hline Mohamed, 2020 & $\begin{array}{c}\text { (24 h, } 3 \text { months, } \\
6 \text { months) of storage in } \\
\text { distilled water at } 37^{\circ} \mathrm{C}\end{array}$ & $\begin{array}{l}\text { Application of MMP } \\
\text { inhibitors }\end{array}$ & Dentin $\mu \mathrm{TBS}$ & $\begin{array}{c}\text { Single Bond Universal } \\
\text { (3M ESPE) }\end{array}$ \\
\hline Paulose, 2017 (b) & $\begin{array}{l}24 \mathrm{~h} \text { and } 1 \text { year of storage in } \\
\text { distilled water at } 37^{\circ} \mathrm{C}\end{array}$ & $\begin{array}{l}\text { Application of MMP } \\
\text { inhibitors }\end{array}$ & Dentin $\mu$ TBS & $\begin{array}{c}\text { Single Bond Universal } \\
\text { (3M ESPE) } \\
\text { Adper Single Bond } 2 \\
\text { (3M ESPE) }\end{array}$ \\
\hline Peng, 2020 & $\begin{array}{c}24 \mathrm{~h} \text { of water storage at } 37^{\circ} \mathrm{C} \\
10,000 \text { cycles between } 5^{\circ} \mathrm{C} \text { and } \\
55^{\circ} \mathrm{C} \\
\text { one-month collagenase ageing } \\
\text { samples were immersed in } 0.1 \\
\mathrm{mg} / \mathrm{mL} \text { collagenase solution } \\
\text { at } 37^{\circ} \mathrm{C}\end{array}$ & $\begin{array}{l}\text { Application of MMP } \\
\text { inhibitors }\end{array}$ & Dentin $\mu$ TBS & $\begin{array}{l}\text { Scotchbond Universal } \\
\text { (SBU; 3M ESPE) }\end{array}$ \\
\hline Shadman, 2018 & $\begin{array}{c}24 \mathrm{~h} \text { and } \\
500 \text { thermal cycles between } \\
5{ }^{\circ} \mathrm{C} \text { and } 55^{\circ} \mathrm{C} \text { in distilled } \\
\text { water at } 37^{\circ} \mathrm{C}\end{array}$ & $\begin{array}{l}\text { Application of MMP } \\
\text { inhibitors }\end{array}$ & Dentin SBS & $\begin{array}{c}\text { Scotchbond Universal } \\
\text { (3M ESPE) } \\
\text { Scotchbond Multi-Purpose } \\
\text { Plus (3M ESPE) }\end{array}$ \\
\hline
\end{tabular}


Table 2. Cont.

\begin{tabular}{|c|c|c|c|c|}
\hline Study & Aging/Storage & Strategy & Bond Strength Test & Adhesive System Used \\
\hline Tekçe, 2016 & $\begin{array}{c}24 \mathrm{~h} \text { of water storage at } 37^{\circ} \mathrm{C} \\
12 \text { months of water storage at } \\
37^{\circ} \mathrm{C}\end{array}$ & $\begin{array}{l}\text { Application of MMP } \\
\text { inhibitors }\end{array}$ & Dentin $\mu \mathrm{TBS}$ & $\begin{array}{l}\text { Single Bond Universal } \\
\text { (3M ESPE) } \\
\text { All Bond Universal } \\
\text { (Bisco Inc.) }\end{array}$ \\
\hline Vivanco, 2020 & $\begin{array}{c}30 \text { days of water storage } \\
\text { at } 37^{\circ} \mathrm{C} \\
1.200 .000 \text { cycles } \\
\text { with } 5 / 37 / 55^{\circ} \mathrm{C}\end{array}$ & $\begin{array}{l}\text { Application of MMP } \\
\text { inhibitors }\end{array}$ & Dentin $\mu$ TBS & $\begin{array}{l}\text { Single Bond Universal } \\
\text { (3M ESPE) } \\
\text { Adper Scotchbond } \\
\text { Multi-Purpose Plus } \\
\text { (3M ESPE) }\end{array}$ \\
\hline Zenobi, 2017 & $\begin{array}{c}\text { water immersion for } 24 \mathrm{~h} \\
200,000 \text { mechanical cycles } \\
\text { under a load of } 30 \mathrm{~N} \text {, at a rate } \\
\text { of } 2 \mathrm{~Hz} \text { for one week }\end{array}$ & $\begin{array}{l}\text { Application of MMP } \\
\text { inhibitors }\end{array}$ & Dentin $\mu$ TBS & $\begin{array}{l}\text { Single Bond Universal } \\
\text { (3M ESPE) }\end{array}$ \\
\hline Ahn, 2014 & $\begin{array}{c}24 \mathrm{~h} \text { of water storage at room } \\
\text { temperature }\end{array}$ & Ethanol-wet bonding & Dentin $\mu \mathrm{TBS}$ & $\begin{array}{c}\text { Clearfil SE Bond (Kuraray } \\
\text { Noritake) } \\
\text { G-aenial Bond (GC Corp) } \\
\text { Xeno V (Dentsply) } \\
\text { BeautiBond (Shofu Inc.) } \\
\text { Adper Easy Bond } \\
\text { (3M ESPE) } \\
\text { Single Bond Universal } \\
\text { (3M ESPE) } \\
\text { All Bond Universal } \\
\text { (Bisco Inc.) }\end{array}$ \\
\hline Ahmed, 2019 & $\begin{array}{c}1 \text { month of water storage at } \\
37^{\circ} \mathrm{C} \\
25,000 \text { and } 50,000 \\
\text { thermocycles }\end{array}$ & Hydrophobic resin layer & Dentin $\mu \mathrm{TBS}$ & $\begin{array}{c}\text { Clearfil SE Bond 2 } \\
\text { (Kuraray Noritake, Osaka, } \\
\text { Japan) } \\
\text { Clearfil Universal Bond } \\
\text { Quick (Kuraray Noritake) } \\
\text { G-Premio Bond (GC Corp., } \\
\text { Tokyo, Japan) } \\
\text { Prime\&Bond Active } \\
\text { (Dentsply, Konstanz, } \\
\text { Germany) }\end{array}$ \\
\hline Chasqueira, 2020 & $\begin{array}{l}24 \mathrm{~h} \text { of water storage at } 37^{\circ} \mathrm{C} \\
6 \text { months in a sodium azide } \\
\text { solution }\left(\mathrm{pH}=7,37^{\circ} \mathrm{C}\right) \\
18 \text { months in a sodium azide } \\
\text { solution }\left(\mathrm{pH}=7,37^{\circ} \mathrm{C}\right)\end{array}$ & Hydrophobic resin layer & Dentin SBS & $\begin{array}{c}\text { Adper Scotchbond 1XT } \\
\text { (3M ESPE) } \\
\text { Clearfil S3 Bond Plus } \\
\text { (Kuraray Noritake) } \\
\text { Solobond M (Voco; } \\
\text { Cuxhaven, Germany) } \\
\text { Adper Easy Bond } \\
\text { (3M ESPE) } \\
\text { Scotchbond Universal } \\
\text { (3M ESPE) }\end{array}$ \\
\hline Ermis, 2019 & $\begin{array}{c}24 \mathrm{~h} \text { of water storage at } 37^{\circ} \mathrm{C} \\
6 \text { months of water storage at } \\
37^{\circ} \mathrm{C}\end{array}$ & Hydrophobic resin layer & Dentin $\mu$ TBS & $\begin{array}{c}\text { Clearfil SE Bond } \\
\text { (Kuraray Noritake) } \\
\text { OptiBond XTR (Kerr Co., } \\
\text { Orange, CA, USA) } \\
\text { Clearfil Universal Bond } \\
\text { Quick (Kuraray Noritake) } \\
\text { Single Bond Universal (3M } \\
\text { ESPE, St Paul, MN, USA) }\end{array}$ \\
\hline Muñoz, 2014 & $24 \mathrm{~h}$ of water storage at $37^{\circ} \mathrm{C}$ & Hydrophobic resin layer & Dentin $\mu \mathrm{TBS}$ & $\begin{array}{l}\text { All Bond Universal (Bisco } \\
\text { Inc., Schaumburg, } \\
\text { IL, USA) } \\
\text { Scotchbond Universal } \\
\text { (3M ESPE) } \\
\text { G-Bond Plus (GC Corp.) }\end{array}$ \\
\hline
\end{tabular}


Table 2. Cont.

\begin{tabular}{|c|c|c|c|c|}
\hline Study & Aging/Storage & Strategy & Bond Strength Test & Adhesive System Used \\
\hline Perdigāo, 2014 & $24 \mathrm{~h}$ of water storage at $37^{\circ} \mathrm{C}$ & Hydrophobic resin layer & $\begin{array}{c}\text { Dentin } \mu \text { TBS } \\
\text { Enamel } \\
\mu \text { SBS }\end{array}$ & G-Bond Plus (GC Corp.) \\
\hline Sezinando, 2015 & $\begin{array}{c}24 \mathrm{~h} \text { of water storage at } 37^{\circ} \mathrm{C} \\
6 \text { months of water storage at } \\
37^{\circ} \mathrm{C}\end{array}$ & Hydrophobic resin layer & Dentin $\mu$ TBS & $\begin{array}{l}\text { Scotchbond Universal } \\
\text { (3M ESPE) } \\
\text { G-Bond Plus (GC Corp.) } \\
\text { All Bond Universal } \\
\text { (Bisco Inc.) }\end{array}$ \\
\hline Vinagre, 2019 & $\begin{array}{c}7 \text { days of water storage at } \\
37^{\circ} \mathrm{C} \\
4 \text { years of water storage at } \\
37^{\circ} \mathrm{C}\end{array}$ & Hydrophobic resin layer & Dentin $\mu$ TBS & $\begin{array}{c}\text { Scotchbond Universal } \\
\text { (3M ESPE) } \\
\text { Adper Scotchbond } \\
\text { Multi-Purpose Plus } \\
\text { (3M ESPE) }\end{array}$ \\
\hline Comba, 2019 & $\begin{array}{c}24 \mathrm{~h} \text { of water storage at } 37^{\circ} \mathrm{C} \\
6 \text { months of water storage at } \\
37^{\circ} \mathrm{C}\end{array}$ & Laser & Dentin $\mu \mathrm{TBS}$ & $\begin{array}{c}\text { Clearfil SE Bond } 2 \\
\text { (Kuraray Noritake) } \\
\text { All Bond Universal } \\
\text { (Bisco Inc.) } \\
\text { OptiBond FL (Kerr Co.) }\end{array}$ \\
\hline Sellan, 2020 & $\begin{array}{l}10,000 \text { cycles between } 5{ }^{\circ} \mathrm{C} \\
\text { and } 55^{\circ} \mathrm{C}\end{array}$ & Laser & Dentin $\mu$ TBS & $\begin{array}{l}\text { Single Bond Universal } \\
\text { (3M ESPE) }\end{array}$ \\
\hline Shadman, 2019 & $\begin{array}{l}500 \text { cycles of thermocycling } \\
\text { between } 5 \text { and } 55^{\circ} \mathrm{C}\end{array}$ & Laser & Dentin SBS & $\begin{array}{l}\text { Scotchbond Universal } \\
\text { (3M ESPE) }\end{array}$ \\
\hline Silva, 2016 & $48 \mathrm{~h}$ of water storage at $37^{\circ} \mathrm{C}$ & Laser & Dentin $\mu$ TBS & $\begin{array}{l}\text { Scotchbond Universal } \\
\text { (3M ESPE) }\end{array}$ \\
\hline Silva, 2019 & $\begin{array}{l}500 \text { cycles of thermocycling } \\
\text { between } 5 \text { and } 55^{\circ} \mathrm{C}\end{array}$ & Laser & Dentin SBS & $\begin{array}{c}\text { Clearfil SE Bond (Kuraray } \\
\text { Noritake) } \\
\text { Scotchbond Universal } \\
\text { (3M ESPE) }\end{array}$ \\
\hline Rechmann, 2017 & $24 \mathrm{~h}$ of water storage at $37^{\circ} \mathrm{C}$ & Laser & $\begin{array}{l}\text { Dentin SBS } \\
\text { Enamel SBS }\end{array}$ & $\begin{array}{c}\text { OptiBond Solo Plus } \\
\text { (Kerr Co.) } \\
\text { Peak Universal Bond } \\
\text { (Ultradent Products Inc., } \\
\text { South Jordan, UT, USA) } \\
\text { Scotchbond Universal } \\
\text { (3M ESPE) }\end{array}$ \\
\hline Trevelin, 2019 & $\begin{array}{c}24 \mathrm{~h} \text { of water storage at } 37^{\circ} \mathrm{C} \\
1 \text { year of storage in artificial } \\
\text { saliva at } 37^{\circ} \mathrm{C}\end{array}$ & Laser & Dentin $\mu$ SBS & $\begin{array}{l}\text { Scotchbond Universal } \\
\text { (3M ESPE) }\end{array}$ \\
\hline Yazici, 2016 & $24 \mathrm{~h}$ of water storage at $37^{\circ} \mathrm{C}$ & Laser & Dentin SBS & $\begin{array}{c}\text { Single Bond Universal } \\
\text { (3M ESPE) }\end{array}$ \\
\hline Chowdhury, 2019 & $24 \mathrm{~h}$ of water storage at $37^{\circ} \mathrm{C}$ & Multiple layer application & Dentin $\mu$ TBS & $\begin{array}{l}\text { Scotchbond Universal } \\
\text { (3M ESPE) } \\
\text { G-Premio Bond (GC Corp) } \\
\text { Clearfil Megabond 2 } \\
\text { (Kuraray Noritake) }\end{array}$ \\
\hline Chowdhury, 2019 (b) & $24 \mathrm{~h}$ of water storage at $37^{\circ} \mathrm{C}$ & Multiple layer application & Dentin $\mu$ TBS & $\begin{array}{c}\text { Scotchbond Universal } \\
\text { (3M ESPE) } \\
\text { G-Premio Bond (GC Corp) } \\
\text { Clearfil Megabond } 2 \\
\text { (Kuraray Noritake) }\end{array}$ \\
\hline Fujiwara, 2018 & $24 \mathrm{~h}$ of water storage at $37^{\circ} \mathrm{C}$ & Multiple layer application & Dentin and Enamel SBS & $\begin{array}{c}\text { Scotchbond Universal } \\
\text { (3M ESPE) } \\
\text { Prime \& Bond elect } \\
\text { (Dentsply, Caulk Milford, } \\
\text { DE, USA) } \\
\text { G-aenial Bond (GC Corp.) } \\
\text { Beautibond (Shofu Inc., } \\
\text { Kyoto, Japan) } \\
\text { OptiBond XTR (Kerr Co.) }\end{array}$ \\
\hline
\end{tabular}


Table 2. Cont.

\begin{tabular}{|c|c|c|c|c|}
\hline Study & Aging/Storage & Strategy & Bond Strength Test & Adhesive System Used \\
\hline Pashaev, 2017 & $\begin{array}{c}24 \mathrm{~h} \text { of water storage at } 37^{\circ} \mathrm{C} \\
6 \text { months of water storage at } \\
37^{\circ} \mathrm{C}\end{array}$ & Multiple layer application & Dentin $\mu$ TBS & $\begin{array}{c}\text { Single Bond Universal } \\
\text { (3M ESPE) } \\
\text { All Bond Universal } \\
\text { (Bisco Inc.) } \\
\text { Adper Easy One } \\
\text { (3M ESPE) } \\
\text { Adper Single Bond } 2 \\
\text { (3M ESPE) }\end{array}$ \\
\hline Taschner, 2014 & $\begin{array}{l}24 \mathrm{~h} \text { in artificial saliva } \\
6 \text { months in artificial saliva at } \\
\qquad 37^{\circ} \mathrm{C} \\
\text { five hours in } 10 \% \mathrm{NaOCl} \text { and } \\
\text { then one hour in distilled } \\
\text { water at room temperature }\end{array}$ & Multiple layer application & Dentin $\mu$ TBS & $\begin{array}{c}\text { iBond SE (Heraeus-Kulzer, } \\
\text { Hanau, Germany) } \\
\text { Xeno V+ (Dentsply DeTrey, } \\
\text { Konstanz, Germany) } \\
\text { Scotchbond Universal } \\
\text { (3M ESPE) } \\
\text { Clearfil S3 Bond } \\
\text { (Kuraray Noritaken) }\end{array}$ \\
\hline Ugurlu, 2020 & $24 \mathrm{~h}$ of water storage at $37^{\circ} \mathrm{C}$ & Multiple layer application & Dentin $\mu$ TBS & $\begin{array}{c}\text { Single Bond Universal } \\
\text { (3M ESPE) } \\
\text { Gluma Bond Universal } \\
\text { (Heraeus Kulzer) } \\
\text { Prime\&Bond Elect } \\
\text { (Dentsply DeTrey) } \\
\text { Clearfil SE Bond } \\
\text { (Kuraray Noritake) }\end{array}$ \\
\hline Zecin-Deren, 2020 & $24 \mathrm{~h}$ of saline storage & Multiple layer application & Dentin SBS & $\begin{array}{c}\text { Single Bond Universal } \\
\text { (3M ESPE) } \\
\text { Prime \& Bond One Select } \\
\text { (Dentsply) } \\
\text { Xeno V (Dentsply) } \\
\text { AdperTM Easy One } \\
\text { (3M ESPE) }\end{array}$ \\
\hline Zecin-Deren, 2020 (b) & $24 \mathrm{~h}$ of saline storage & Multiple layer application & Dentin SBS & $\begin{array}{c}\text { Single Bond Universal } \\
\text { (3M ESPE) } \\
\text { Prime \& Bond One Select } \\
\text { (Dentsply) } \\
\text { Xeno V (Dentsply) } \\
\text { AdperTM Easy One } \\
\text { (3M ESPE) }\end{array}$ \\
\hline Ayres, 2017 & $\begin{array}{c}24 \mathrm{~h} \text { of water storage at } 37^{\circ} \mathrm{C} \\
2 \text { years of water storage } \\
\text { at } 37^{\circ} \mathrm{C}\end{array}$ & $\begin{array}{l}\text { Non-thermal atmospheric } \\
\text { plasma }\end{array}$ & Dentin $\mu$ TBS & $\begin{array}{l}\text { Scotchbond Universal } \\
\text { (3M ESPE) }\end{array}$ \\
\hline Ayres, 2018 & $\begin{array}{c}24 \mathrm{~h} \text { of water storage at } 37^{\circ} \mathrm{C} \\
1 \text { years of direct water } \\
\text { exposure } \\
1 \text { year of simulated pulpal } \\
\text { pressure }\end{array}$ & $\begin{array}{l}\text { Non-thermal atmospheric } \\
\text { plasma }\end{array}$ & Dentin $\mu$ TBS & $\begin{array}{l}\text { Scotchbond Universal } \\
\text { (3M ESPE) }\end{array}$ \\
\hline Alqahtani, 2014 & $24 \mathrm{~h}$ of water storage at $37^{\circ} \mathrm{C}$ & Prolonged curing time & Dentin SBS & $\begin{array}{c}\text { Single Bond Universal } \\
\text { (3M ESPE) } \\
\text { Xeno V+ (Dentsply) } \\
\text { AdheSE One F VivaPen } \\
\text { (Ivoclar Vivadent) } \\
\end{array}$ \\
\hline Sampaio, 2017 & $\begin{array}{c}24 \mathrm{~h} \text { of water storage at } 37^{\circ} \mathrm{C} \\
2 \text { years of water storage } \\
\text { at } 37^{\circ} \mathrm{C}\end{array}$ & Prolonged curing time & Dentin $\mu$ TBS & $\begin{array}{c}\text { Adper Single Bond } 2 \\
\text { (3M ESPE) } \\
\text { Optibond Solo Plus (Kerr) } \\
\text { Optibond All-In-One } \\
\text { (Kerr) } \\
\text { Clearfil SE Bond } \\
\text { (Kuraray Noritake) } \\
\text { Scotchbond Universal } \\
\text { (3M ESPE) }\end{array}$ \\
\hline
\end{tabular}


Table 2. Cont

\begin{tabular}{|c|c|c|c|c|}
\hline Study & Aging/Storage & Strategy & Bond Strength Test & Adhesive System Used \\
\hline $\mathrm{Fu}, 2017$ & $24 \mathrm{~h}$ of water storage at $37^{\circ} \mathrm{C}$ & Prolonged blowing time & Dentin $\mu$ TBS & $\begin{array}{c}\text { Scotchbond Universal } \\
\text { (3M ESPE) } \\
\text { All Bond Universal } \\
\text { (Bisco Inc.) } \\
\text { G-Premio Bond (GC Corp) } \\
\text { Clearfil Universal Bond } \\
\text { (Kuraray Noritake) } \\
\text { Optibond All-in-one } \\
\text { (Kerr) }\end{array}$ \\
\hline Luque-Martinez, 2014 & $24 \mathrm{~h}$ of water storage at $37^{\circ} \mathrm{C}$ & Prolonged blowing time & Dentin $\mu$ TBS & $\begin{array}{l}\text { Scotchbond Universal } \\
\text { (3M ESPE) } \\
\text { All Bond Universal } \\
\text { (Bisco Inc.) } \\
\text { Prime \& Bond Elect } \\
\text { (Dentsply) }\end{array}$ \\
\hline Saikaew, 2018 & $\begin{array}{c}24 \mathrm{~h} \text { of water storage at } 37^{\circ} \mathrm{C} \\
1 \text { year of water storage }\end{array}$ & Prolonged blowing time & Dentin $\mu$ TBS & $\begin{array}{c}\text { Scotchbond Universal } \\
\text { (3M ESPE) } \\
\text { G-Premio Bond (GC Corp) } \\
\text { Clearfil Universal Bond } \\
\text { (Kuraray Noritake) }\end{array}$ \\
\hline Irmak, 2018 & $\begin{array}{c}24 \mathrm{~h} \text { of water storage at } 37^{\circ} \mathrm{C} \\
10,000 \text { cycles between } 5^{\circ} \mathrm{C} \text { and } \\
55^{\circ} \mathrm{C}\end{array}$ & Scrubbing technique & Dentin $\mu$ TBS & $\begin{array}{c}\text { Single Bond Universal } \\
\text { (3M ESPE) } \\
\text { Clearfil Universal Bond } \\
\text { Quick (Kuraray Noritake) }\end{array}$ \\
\hline Jang, 2018 & $\begin{array}{l}24 \mathrm{~h} \text { of water storage at } 37^{\circ} \mathrm{C} \\
\text { Artificial aging ( } 10 \% \text { aqueous } \\
\text { sodium hypochlorite solution } \\
\text { for } 1 \mathrm{~h} \text { at room temperature) }\end{array}$ & Scrubbing technique & Dentin $\mu \mathrm{TBS}$ & G-Premio Bond (GC Corp) \\
\hline Thanatvarakorn, 2016 & $24 \mathrm{~h}$ of water storage at $37^{\circ} \mathrm{C}$ & Scrubbing technique & Dentin $\mu \mathrm{TBS}$ & $\begin{array}{l}\text { SE One (Kuraray Noritake) } \\
\text { Scotchbond Universal } \\
\text { (3M ESPE) }\end{array}$ \\
\hline Kharouf, 2019 & $24 \mathrm{~h}$ of water storage at $37^{\circ} \mathrm{C}$ & Selective dentin etching & Dentin $\mu \mathrm{TBS}$ & $\begin{array}{l}\text { Prime \& Bond }{ }^{\circledR} \text { active } \\
\text { (Dentsply) }\end{array}$ \\
\hline Stape, 2018 & $\begin{array}{c}24 \mathrm{~h} \text { of water storage at } 37^{\circ} \mathrm{C} \\
6 \text { months of storage in artificial } \\
\text { saliva }\end{array}$ & Selective dentin etching & Dentin $\mu$ TBS & $\begin{array}{c}\text { Scotchbond Universal } \\
\text { (3M ESPE) } \\
\text { Scotchbond Multi-Purpose } \\
\text { Plus (3M ESPE) }\end{array}$ \\
\hline Takamizawa, 2016 & $24 \mathrm{~h}$ of water storage at $37^{\circ} \mathrm{C}$ & Selective dentin etching & Dentin SBS & $\begin{array}{c}\text { Prime \& Bond Elect } \\
\text { (Dentsply) } \\
\text { Scotchbond Universal } \\
\text { (3M ESPE) } \\
\text { G-ænial Bond (GC Corp.) } \\
\text { OptiBond XTR (Kerr Co.) }\end{array}$ \\
\hline Pashaev,2017 & $\begin{array}{c}24 \mathrm{~h} \text { of water storage at } 37^{\circ} \mathrm{C} \\
6 \text { months of water storage at } \\
37^{\circ} \mathrm{C}\end{array}$ & $\begin{array}{l}\text { Prolonged application } \\
\text { time }\end{array}$ & Dentin $\mu$ TBS & $\begin{array}{c}\text { All Bond Universal } \\
\text { (Bisco Inc.) } \\
\text { Single Bond Universal } \\
\text { (3M ESPE) } \\
\text { Adper Easy One (3M } \\
\text { ESPE, St Paul, MN, USA) } \\
\text { Adper Single Bond 2 (3M } \\
\text { ESPE, St Paul, MN, USA) }\end{array}$ \\
\hline Ahmed, 2019 & $\begin{array}{c}1 \text { week of water storage at } 37 \\
6 \text { months of water storage at } \\
37^{\circ} \mathrm{C}\end{array}$ & $\begin{array}{l}\text { Prolonged application } \\
\text { time }\end{array}$ & Dentin $\mu \mathrm{TBS}$ & $\begin{array}{c}\text { Scotchbond Universal } \\
\text { (3M ESPE) } \\
\text { Clearfil Universal Bond } \\
\text { Quick (Kuraray Noritake) } \\
\text { Clearfil SE Bond 2 } \\
\text { (Kuraray Noritake) }\end{array}$ \\
\hline
\end{tabular}


Table 2. Cont.

\begin{tabular}{|c|c|c|c|c|}
\hline Study & Aging/Storage & Strategy & Bond Strength Test & Adhesive System Used \\
\hline Amsler, 2015 & $24 \mathrm{~h}$ of water storage at $37^{\circ} \mathrm{C}$ & $\begin{array}{c}\text { Shortened application } \\
\text { time }\end{array}$ & Dentin SBS & $\begin{array}{c}\text { Syntac Classic (Ivoclar } \\
\text { Vivadent; Schaan, } \\
\text { Liechtenstein) } \\
\text { OptiBond FL (Kerry) } \\
\text { Clearfil SE Bond } \\
\text { (Kuraray Noritake) } \\
\text { AdheSE (Ivoclar Vivadent) } \\
\text { Xeno Select (Dentsply) } \\
\text { Scotchbond Universal } \\
\text { (3M ESPE) }\end{array}$ \\
\hline Huang, 2017 & $\begin{array}{l}24 \mathrm{~h} \text { of water storage at } 37^{\circ} \mathrm{C} \\
10,000 \text { thermal cycles } \\
\left(10^{\circ} \mathrm{C} \text { for one min, } 25^{\circ} \mathrm{C} \text { for }\right. \\
\text { one min and } 55^{\circ} \mathrm{C} \text { for one } \\
\text { min) and } 240,000 \text { mechanical } \\
\text { cycles }\end{array}$ & $\begin{array}{c}\text { Shortened application } \\
\text { time }\end{array}$ & Dentin $\mu$ TBS & G-Premio Bond (GC Corp) \\
\hline Saikaew, 2016 & $24 \mathrm{~h}$ of water storage at $37^{\circ} \mathrm{C}$ & $\begin{array}{c}\text { Shortened application } \\
\text { time }\end{array}$ & Dentin $\mu \mathrm{TBS}$ & $\begin{array}{c}\text { G-Premio Bond (GC Corp) } \\
\text { Clearfil Universal Bond } \\
\text { (Kuraray Noritake) } \\
\text { Scotchbond Universal } \\
\text { (3M ESPE) }\end{array}$ \\
\hline Saikaew, 2018 & $\begin{array}{l}24 \mathrm{~h} \text { of water storage at } 37^{\circ} \mathrm{C} \\
1 \text { year of water storage at } 37^{\circ} \mathrm{C}\end{array}$ & $\begin{array}{c}\text { Shortened application } \\
\text { time }\end{array}$ & Dentin $\mu \mathrm{TBS}$ & $\begin{array}{c}\text { G-Premio Bond (GC Corp) } \\
\text { Clearfil Universal Bond } \\
\text { (Kuraray Noritake) } \\
\text { Scotchbond Universal } \\
\text { (3M ESPE) }\end{array}$ \\
\hline Zecin-Deren, 2019 & $24 \mathrm{~h}$ saline solution & $\begin{array}{l}\text { Shortened application } \\
\text { time }\end{array}$ & Dentin SBS & $\begin{array}{c}\text { Single Bond Universal } \\
\text { (3M ESPE) } \\
\text { Prime \& Bond One Select } \\
\text { (Dentsply) } \\
\text { Xeno V (Dentsply) } \\
\text { AdperTM Easy One } \\
\text { (3M ESPE) }\end{array}$ \\
\hline Guarda, 2020 & $24 \mathrm{~h}$ of water storage at $37^{\circ} \mathrm{C}$ & $\begin{array}{l}\text { Electric-current } \\
\text { application }\end{array}$ & Dentin $\mu$ TBS & $\begin{array}{c}\text { Single Bond Universal } \\
\text { (3M ESPE) } \\
\text { Adper Single Bond } 2 \\
\text { (3M ESPE) } \\
\text { Clearfil SE Bond } \\
\text { (Kuraray Noritake) }\end{array}$ \\
\hline Cecchin, 2018 & $24 \mathrm{~h}$ of water storage at $37^{\circ} \mathrm{C}$ & $\begin{array}{l}\text { a-hydroxy glycolic acid } \\
\text { (GA) as a surface } \\
\text { pretreatment }\end{array}$ & Dentin and enamel $\mu$ TBS & $\begin{array}{l}\text { Scotchbond Universal } \\
\text { (3M ESPE) } \\
\text { Adper Single Bond } \\
\text { (3M ESPE) } \\
\text { One Step Plus Bisco } \\
\text { (Schaumburg) }\end{array}$ \\
\hline Cangul, 2020 & $24 \mathrm{~h}$ of water storage at $37^{\circ} \mathrm{C}$ & $\begin{array}{l}\text { Ozone as cavity } \\
\text { disinfectant }\end{array}$ & Dentin SBS & $\begin{array}{c}\text { Clearfil SE Protect primer } \\
\text { (Kuraray) } \\
\text { Clearfil SE Protect Bond } \\
\text { (Kuraray) } \\
\text { Peek Universal (Ultradent } \\
\text { Products Inc.) } \\
\text { Gluma 2 Bond } \\
\text { (Heraeus Kulzer) }\end{array}$ \\
\hline
\end{tabular}


Table 2. Cont.

\begin{tabular}{|c|c|c|c|c|}
\hline Study & Aging/Storage & Strategy & Bond Strength Test & Adhesive System Used \\
\hline Demirel, 2019 & $\begin{array}{l}\text { 10,000 thermocycles between } \\
55^{\circ} \mathrm{C} \text { and } 55^{\circ} \mathrm{C}\end{array}$ & $\begin{array}{l}\text { Different etching modes } \\
\text { and etching time }\end{array}$ & Dentin $\mu$ SBS & $\begin{array}{c}\text { Single Bond Universal } \\
\text { (3M ESPE) } \\
\text { All Bond Universal } \\
\text { (Bisco Inc.) } \\
\text { Clearfil Universal Bond } \\
\text { Quick (Kuraray Noritake) } \\
\text { Clearfil SE Bond (Kuraray } \\
\text { Noritake) } \\
\text { Clearfil S3 Bond Plus } \\
\text { (Kuraray Noritake) } \\
\text { Adper Single Bond 2 } \\
\text { (3M ESPE) }\end{array}$ \\
\hline Akarsu, 2019 & $2 \mathrm{~h}$ of water storage at $36^{\circ} \mathrm{C}$ & $\begin{array}{l}\text { Pre-warming of adhesive } \\
\text { bottles }\end{array}$ & Dentin SBS & $\begin{array}{l}\text { All Bond Universal } \\
\text { (Bisco Inc.) } \\
\text { Single Bond Universal } \\
\text { (3M ESPE) } \\
\text { Clearfil SE Bond } \\
\text { (Kuraray Noritake) }\end{array}$ \\
\hline Chen, 2020 & $\begin{array}{c}24 \mathrm{~h} \text { of water storage at } 37^{\circ} \mathrm{C} \\
100 \text { days of water storage } \\
\text { at } 37^{\circ} \mathrm{C}\end{array}$ & $\begin{array}{l}\text { Air-blowing with warm } \\
\text { air }\end{array}$ & Dentin $\mu \mathrm{TBS}$ & $\begin{array}{c}\text { All Bond Universal } \\
\text { (Bisco Inc.) } \\
\text { Single Bond Universal } \\
\text { (3M ESPE) } \\
\text { Clearfil Universal Bond } \\
\text { (Kuraray Noritake) } \\
\text { Gluma Bond Universal } \\
\text { (Heraeus Kulzer) } \\
\text { Adhese Universal (Ivoclar } \\
\text { Vivadent) }\end{array}$ \\
\hline Thanatvarakorn, 2018 & $24 \mathrm{~h}$ of water storage at $37^{\circ} \mathrm{C}$ & $\begin{array}{l}\text { Smear layer } \\
\text { deproteinizing }\end{array}$ & Dentin $\mu \mathrm{TBS}$ & $\begin{array}{c}\text { SE One (Kuraray Noritake) } \\
\text { Scotchbond Universal } \\
\text { (3M ESPE) } \\
\text { BeautiBond Multi (Shofu) } \\
\text { Bond Force } \\
\text { (Tokuyama Dental) }\end{array}$ \\
\hline
\end{tabular}

Separate analysis for each technique was performed. The main results of the datasets evaluated are shown in Figures 2-16. The bond strength of universal adhesives to dentin was improved when the following techniques were used: Previous application of MMP inhibitors $(p<0.001)$, prolonged application time $(p=0.007)$, scrubbing technique $(p<0.001)$, selective dentin etching $(p<0.001)$, non-atmospheric plasma $(p=0.01)$, ethanol-wet bonding $(p<0.01)$, prolonged blowing time $(p=0.02)$, multiple layer application $(p<0.001)$, prolonged curing time $(p=0.006)$, and hydrophobic layer coating $(p<0.001)$.

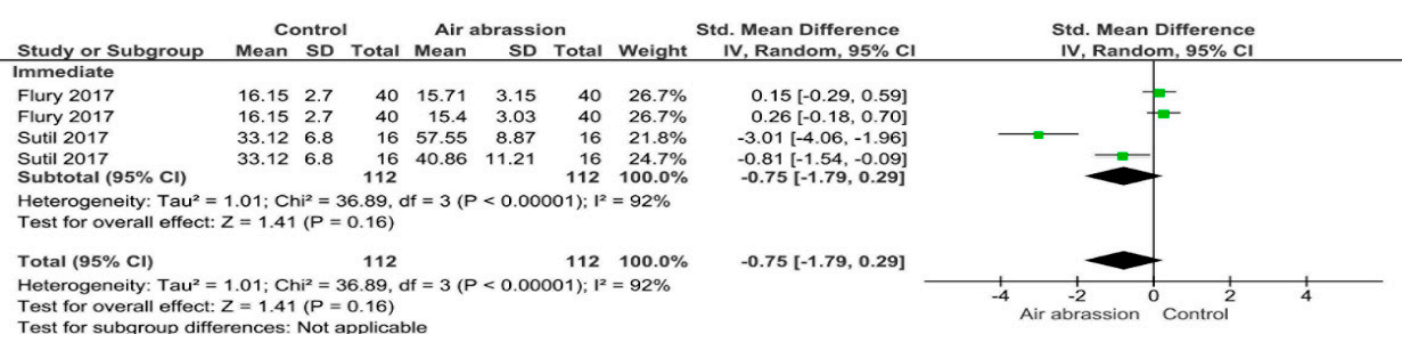

Figure 2. Results of the meta-analysis of immediate and aged bond strength of universal adhesive to dentin after air abrasion. 


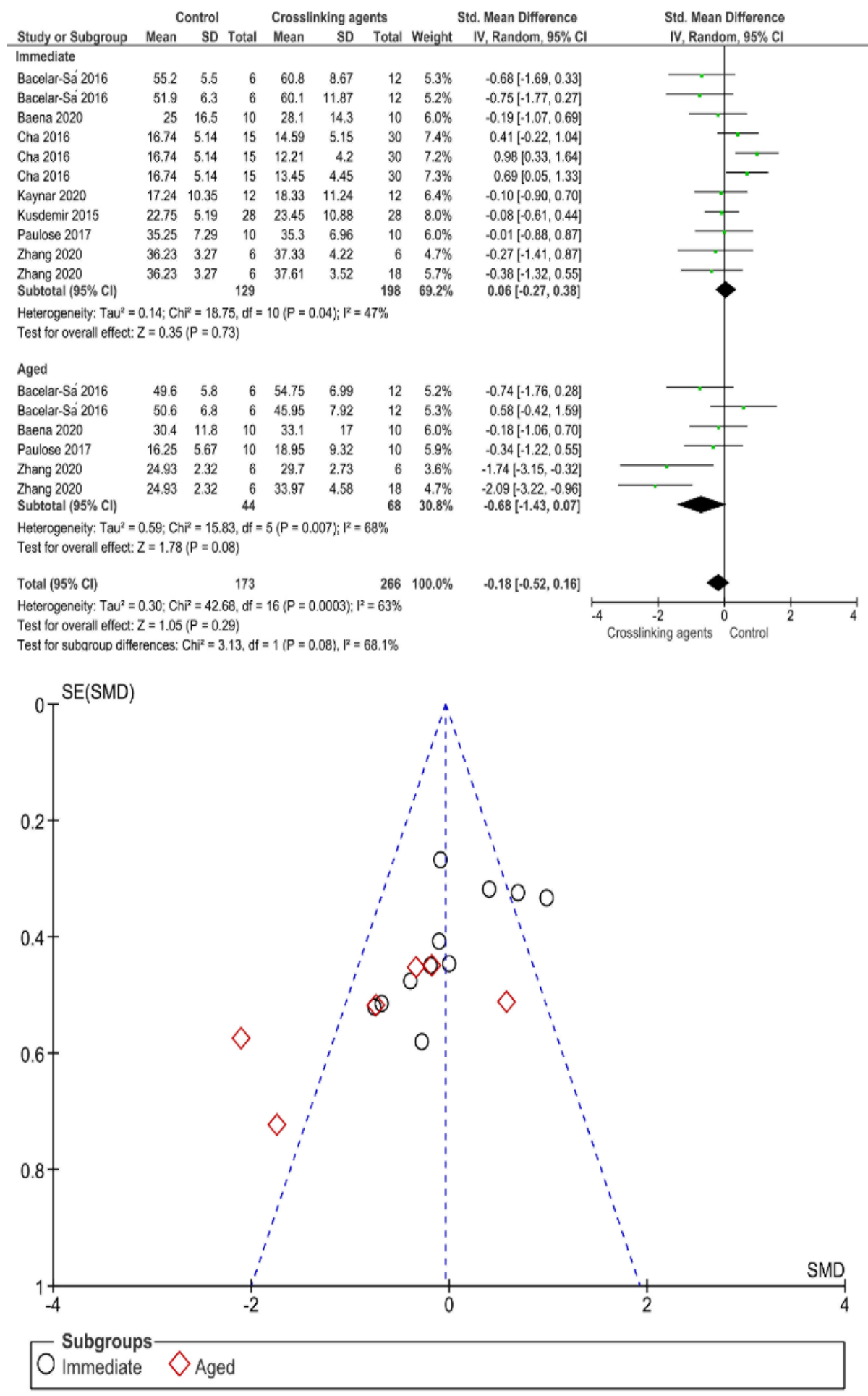

Figure 3. Results of the meta-analysis of immediate and aged bond strength of universal adhesive to dentin after application of crosslinking agents. 

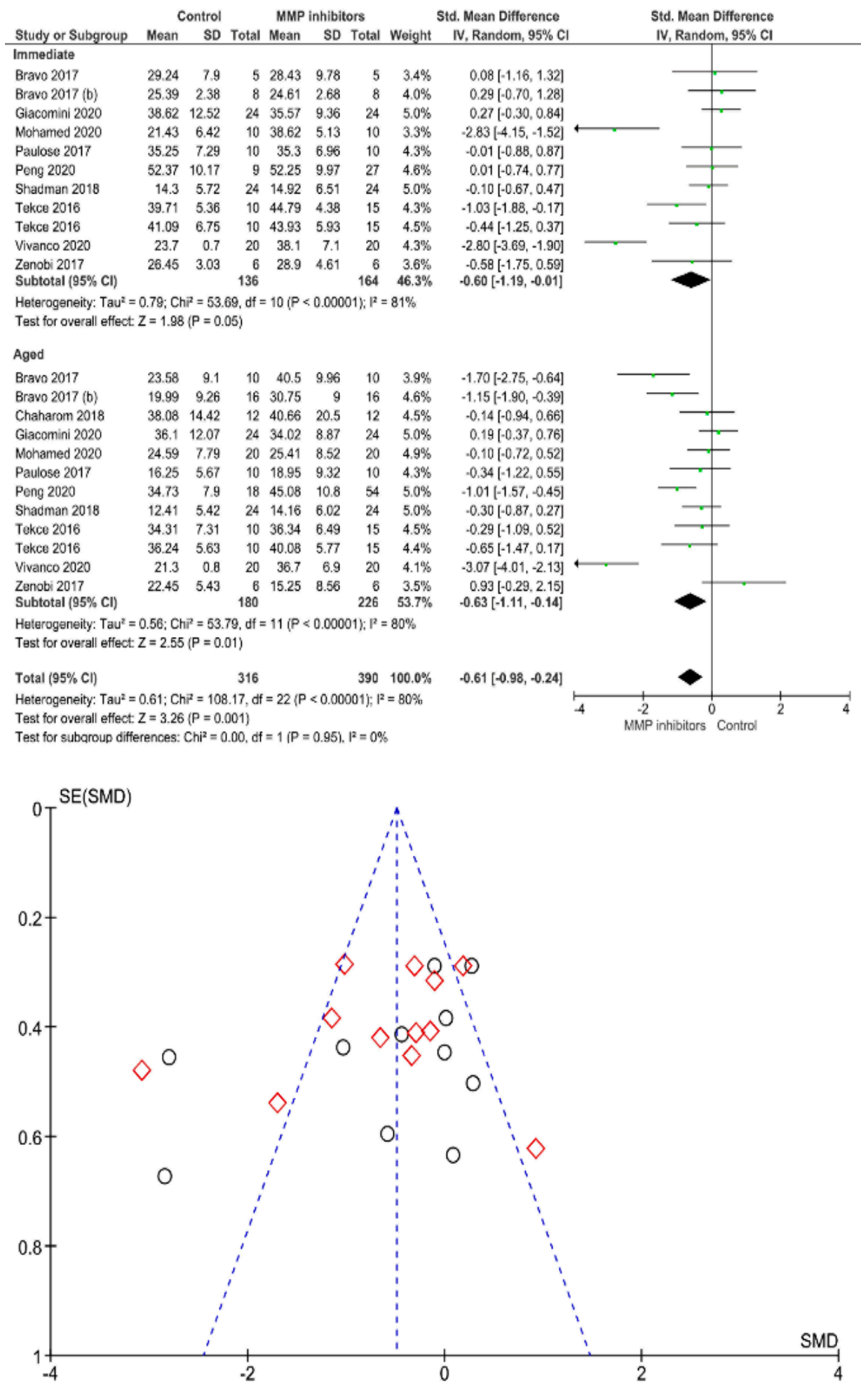

${ }^{\circ}$ Immediate $\diamond$ Aged

Figure 4. Results of the meta-analysis of immediate and aged bond strength of universal adhesive to dentin after application of MMP inhibitors. 


\begin{tabular}{|c|c|c|c|c|c|c|c|c|c|c|c|}
\hline \multirow{3}{*}{$\begin{array}{l}\text { Study or Subgroup } \\
\text { Immediate }\end{array}$} & \multicolumn{3}{|c|}{ Control } & \multicolumn{3}{|c|}{ Dentin desensitizer } & \multicolumn{2}{|r|}{ Std. Mean Difference } & \multirow{2}{*}{\multicolumn{2}{|c|}{$\begin{array}{l}\text { Std. Mean Difference } \\
\text { IV, Random, } 95 \% \mathrm{CI}\end{array}$}} & \\
\hline & \multirow[t]{2}{*}{ Mean } & \multirow[t]{2}{*}{ SD } & \multirow{2}{*}{ Total } & \multirow[t]{2}{*}{ Mean } & \multirow[t]{2}{*}{ SD } & \multirow[t]{2}{*}{ Total } & \multirow{2}{*}{ Weight } & \multirow[t]{2}{*}{ IV, Random, $95 \% \mathrm{Cl}$} & & & \\
\hline & & & & & & & & & & & \\
\hline Luong 2020 & 31.4 & 7.99 & 20 & 24.85 & 7.29 & 20 & $31.8 \%$ & $0.84[0.19,1.49]$ & & & \\
\hline $\begin{array}{l}\text { Luong } 2020 \\
\text { Subtotal }(95 \% \mathrm{Cl})\end{array}$ & 31.4 & 7.99 & $\begin{array}{l}20 \\
40\end{array}$ & 28.65 & 6.07 & $\begin{array}{l}20 \\
40\end{array}$ & $\begin{array}{l}32.3 \% \\
64.1 \%\end{array}$ & $\begin{array}{l}0.38[-0.25,1.01] \\
0.60[0.15,1.05]\end{array}$ & & & \\
\hline $\begin{array}{l}\text { Heterogeneity: } \mathrm{Tau}^{2}= \\
\text { Test for overall effect: }\end{array}$ & $\begin{array}{l}0.00 ; \mathrm{Ch} \\
\mathrm{Z}=2.61\end{array}$ & $\begin{array}{l}\mathrm{i}^{2}=1 . C \\
(P=0\end{array}$ & $\begin{array}{l}00, \mathrm{df}= \\
.009)\end{array}$ & $1(P=0$ & 2); $1^{2}=$ & & & & & & \\
\hline Aged & & & & & & & & & & & \\
\hline $\begin{array}{l}\text { Siso } 2016 \\
\text { Subtotal }(95 \% \mathrm{Cl})\end{array}$ & 24.77 & 7.74 & $\begin{array}{l}46 \\
46\end{array}$ & 13.98 & 5.54 & $\begin{array}{l}46 \\
46\end{array}$ & $\begin{array}{l}35.9 \% \\
35.9 \%\end{array}$ & $\begin{array}{l}1.59[1.12,2.06] \\
1.59[1.12,2.06]\end{array}$ & & & \\
\hline $\begin{array}{l}\text { Heterogeneity: Not ap } \\
\text { Test for overall effect: }\end{array}$ & $\begin{array}{l}\text { plicable } \\
Z=6.61\end{array}$ & $(P<0$ & .00001 & & & & & & & & \\
\hline Total $(95 \% \mathrm{Cl})$ & & & 86 & & & 86 & $100.0 \%$ & $0.96[0.22,1.70]$ & & & \\
\hline $\begin{array}{l}\text { Heterogeneity: } \mathrm{Tau}^{2}= \\
\text { Test for overall effect: } \\
\text { Test for subaroup diff }\end{array}$ & $\begin{array}{l}0.34 ; \mathrm{Ch} \\
\mathrm{Z}=2.54 \\
\text { rences: }\end{array}$ & $\begin{array}{l}\mathrm{i}^{2}=9.8 \\
(\mathrm{P}=0 \\
\mathrm{Chi}^{2}=\end{array}$ & $\begin{array}{l}\text { 82, df = } \\
.01) \\
8.83, d f\end{array}$ & $2(P=0$ & $07) ; 1^{2}=$ & & & & $\begin{array}{cc}-4 & -2 \\
& \text { Dentin desensitizer }\end{array}$ & Control & 2 \\
\hline
\end{tabular}

Figure 5. Results of the meta-analysis of immediate and aged bond strength of universal adhesive to dentin after application of dentin desensitizers.

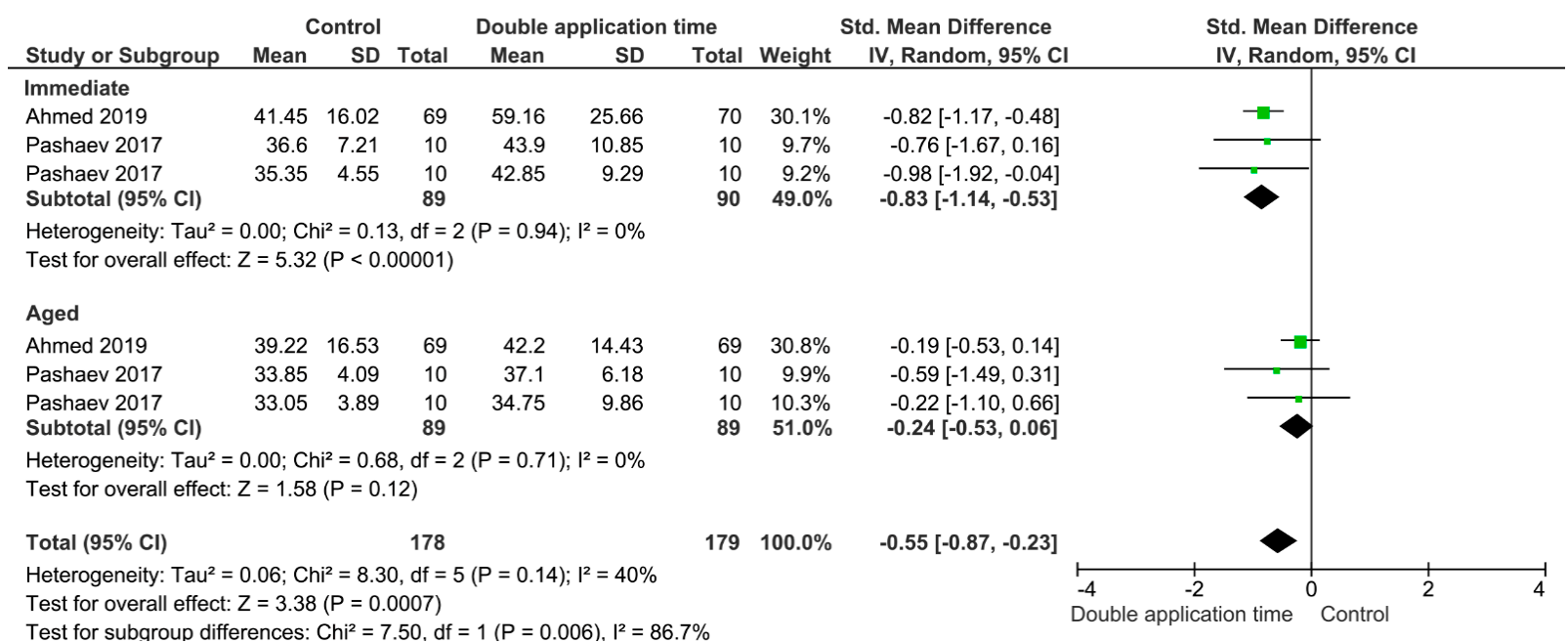

Figure 6. Results of the meta-analysis of immediate and aged bond strength of universal adhesive to dentin using the double application time technique.

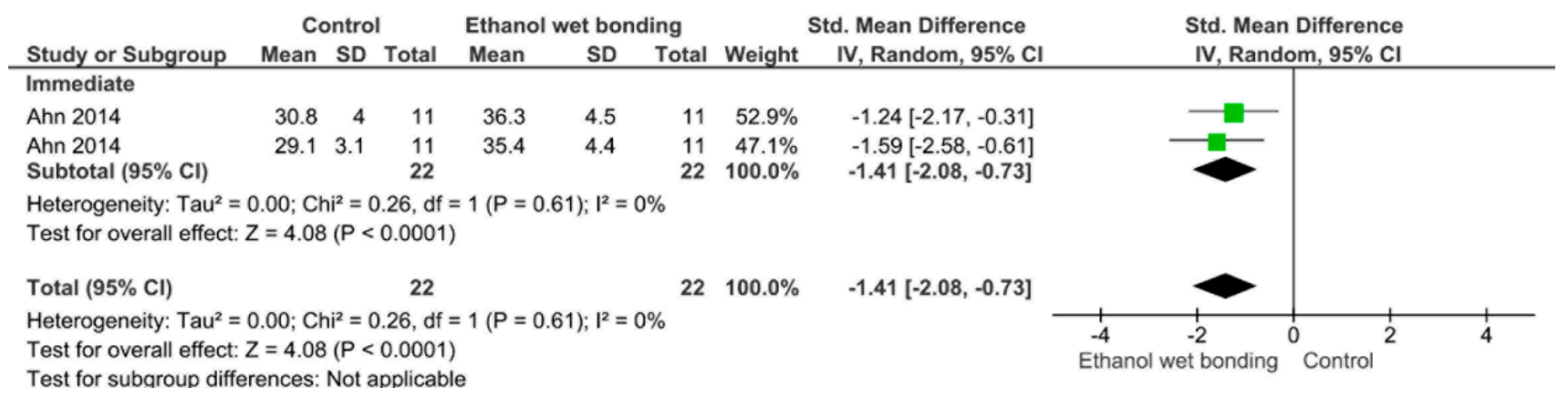

Figure 7. Results of the meta-analysis of immediate bond strength of universal adhesive to dentin using the ethanol-wet bonding technique. 

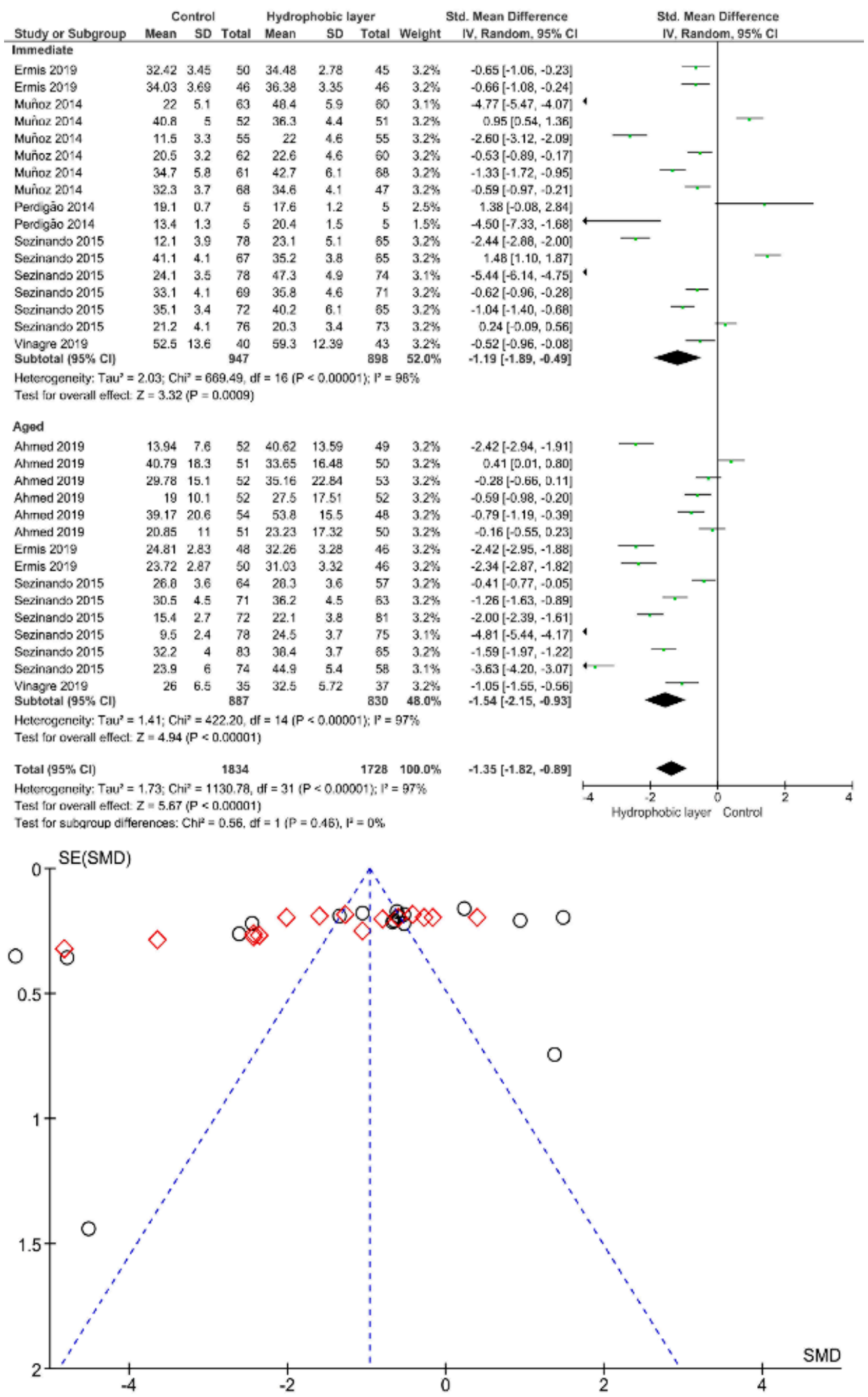

O Immediate $\diamond$ Aged

Figure 8. Results of the meta-analysis of immediate and aged bond strength of universal adhesive to dentin after the application of a hydrophobic layer. 


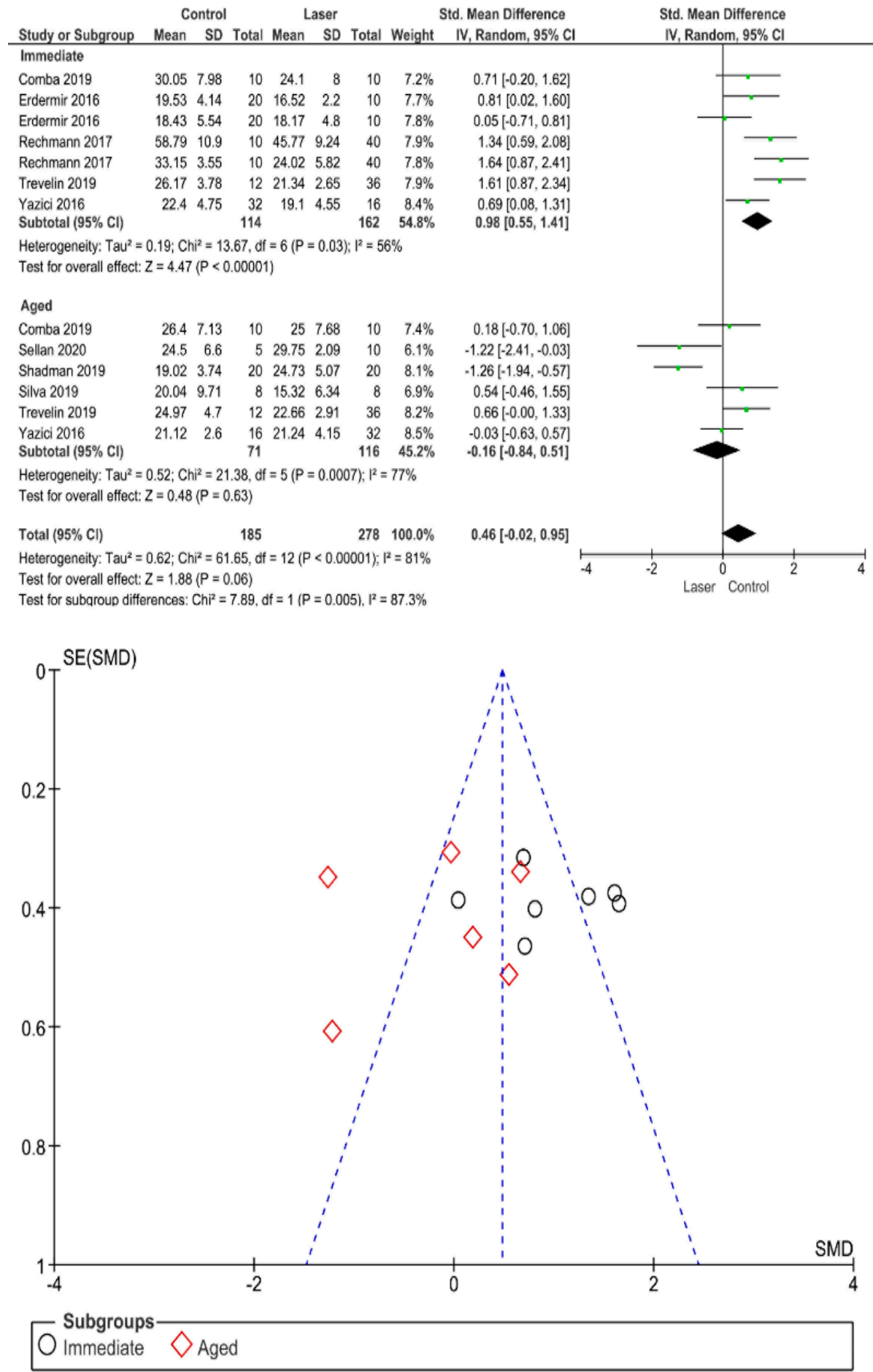

Figure 9. Results of the meta-analysis of immediate and aged bond strength of universal adhesive to dentin using a laser as surface pretreatment. 


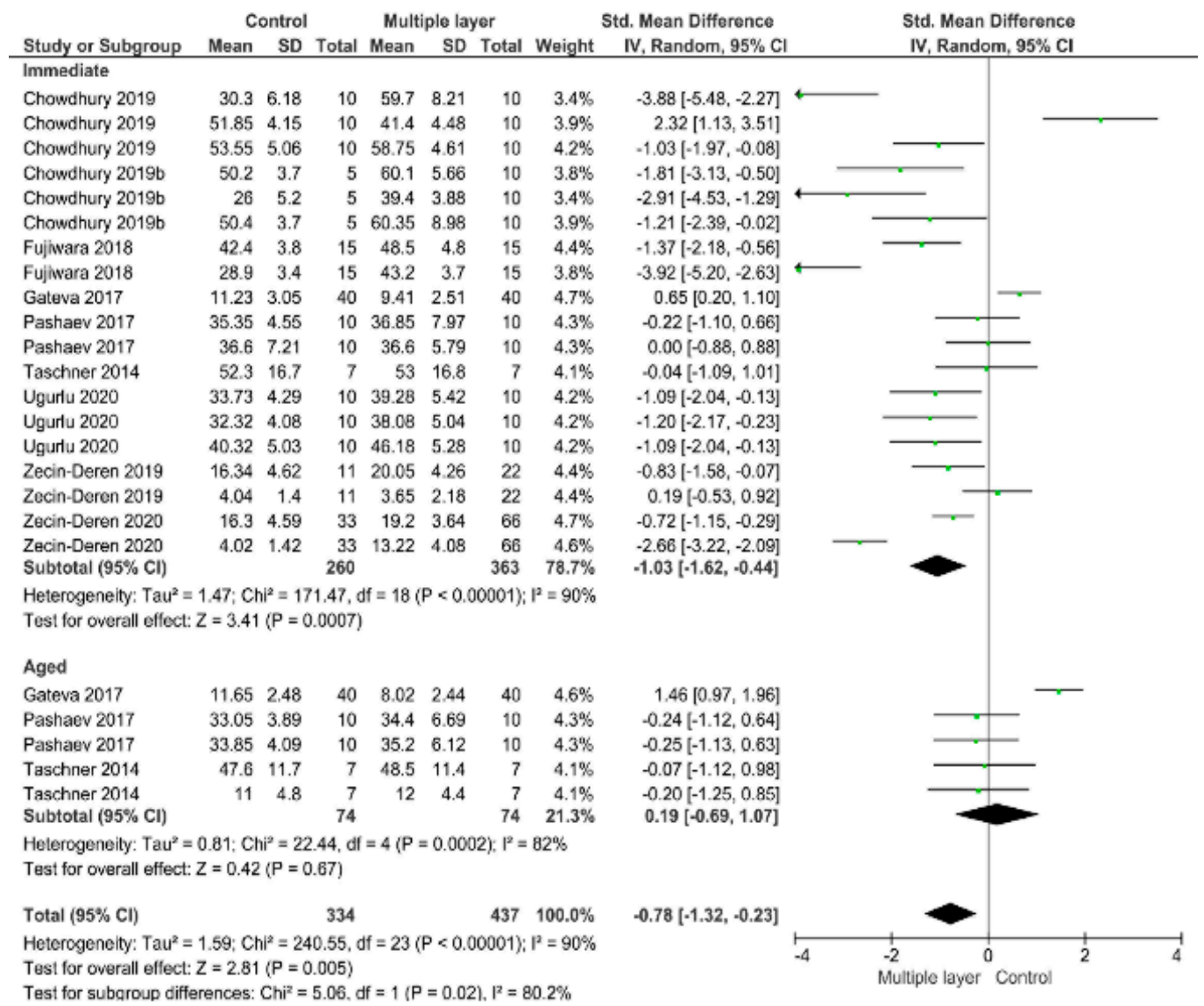

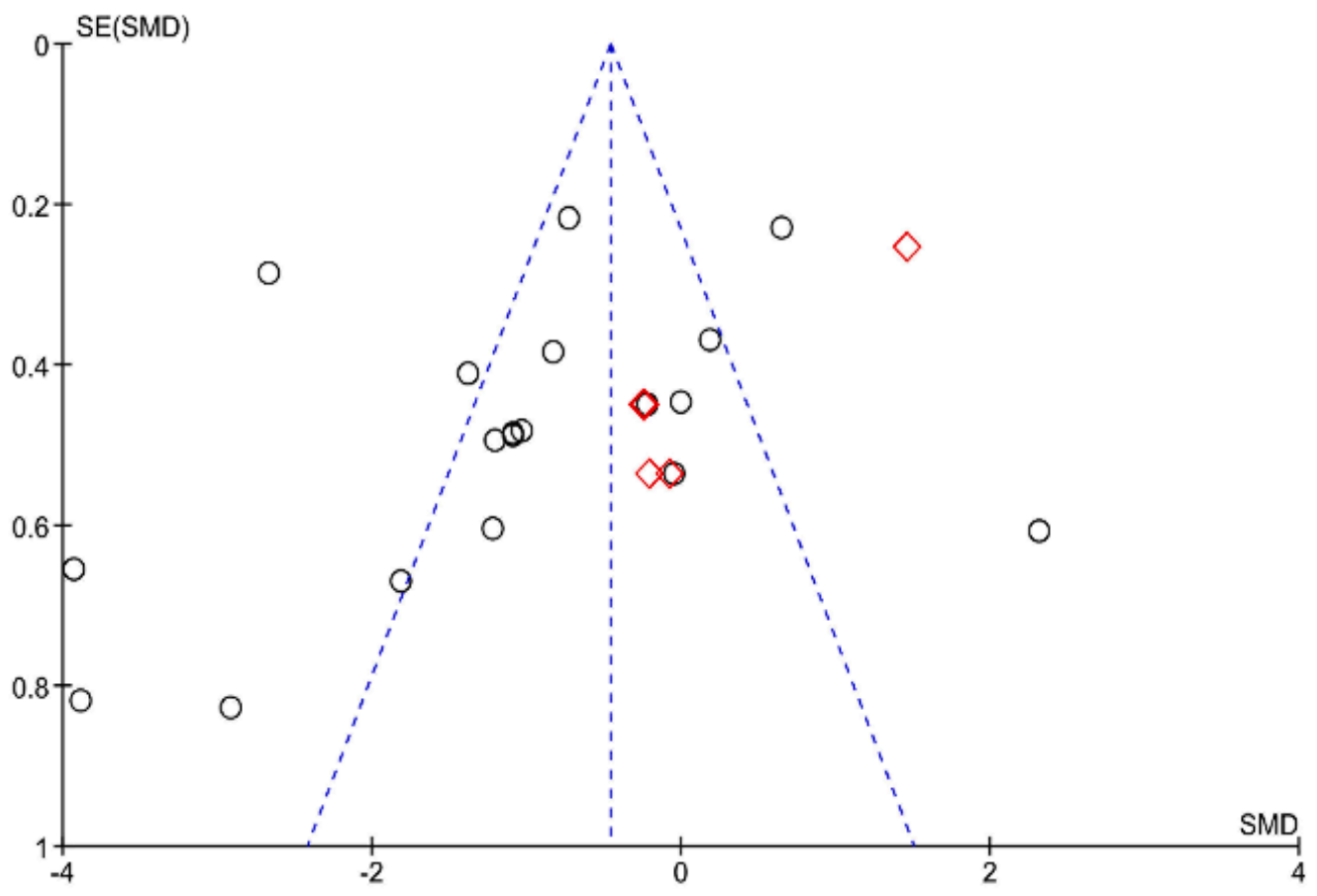

O Immediate $\diamond$ Aged

Figure 10. Results of the meta-analysis of immediate and aged bond strength of universal adhesive to dentin used in multiple layers. 


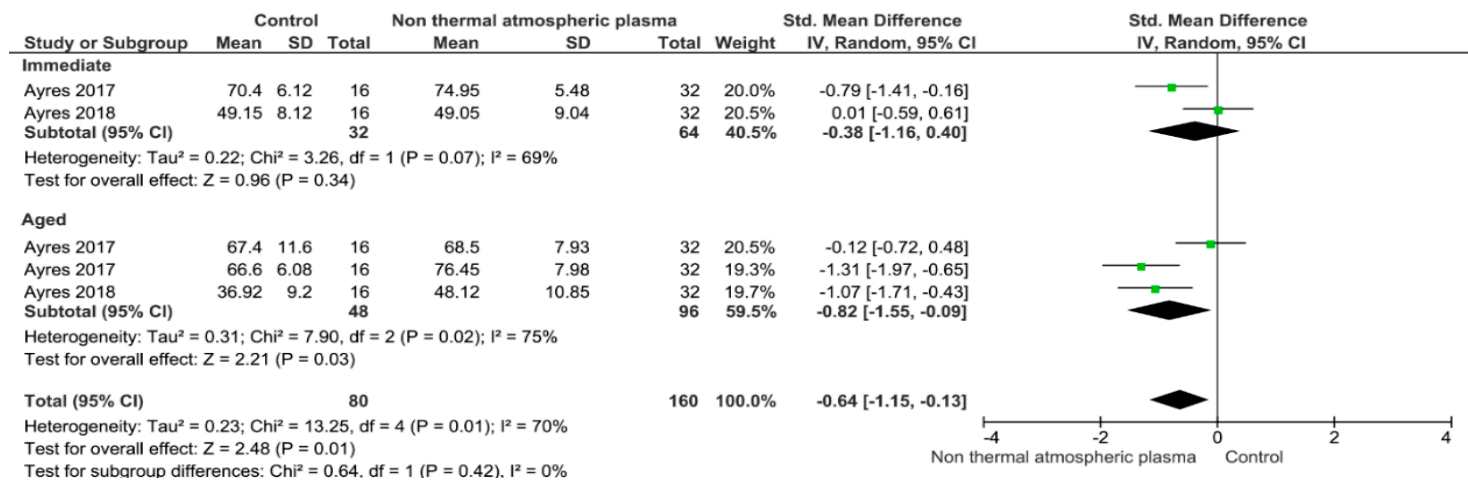

Figure 11. Results of the meta-analysis of immediate and aged bond strength of universal adhesive to dentin using non-atmospheric plasma.
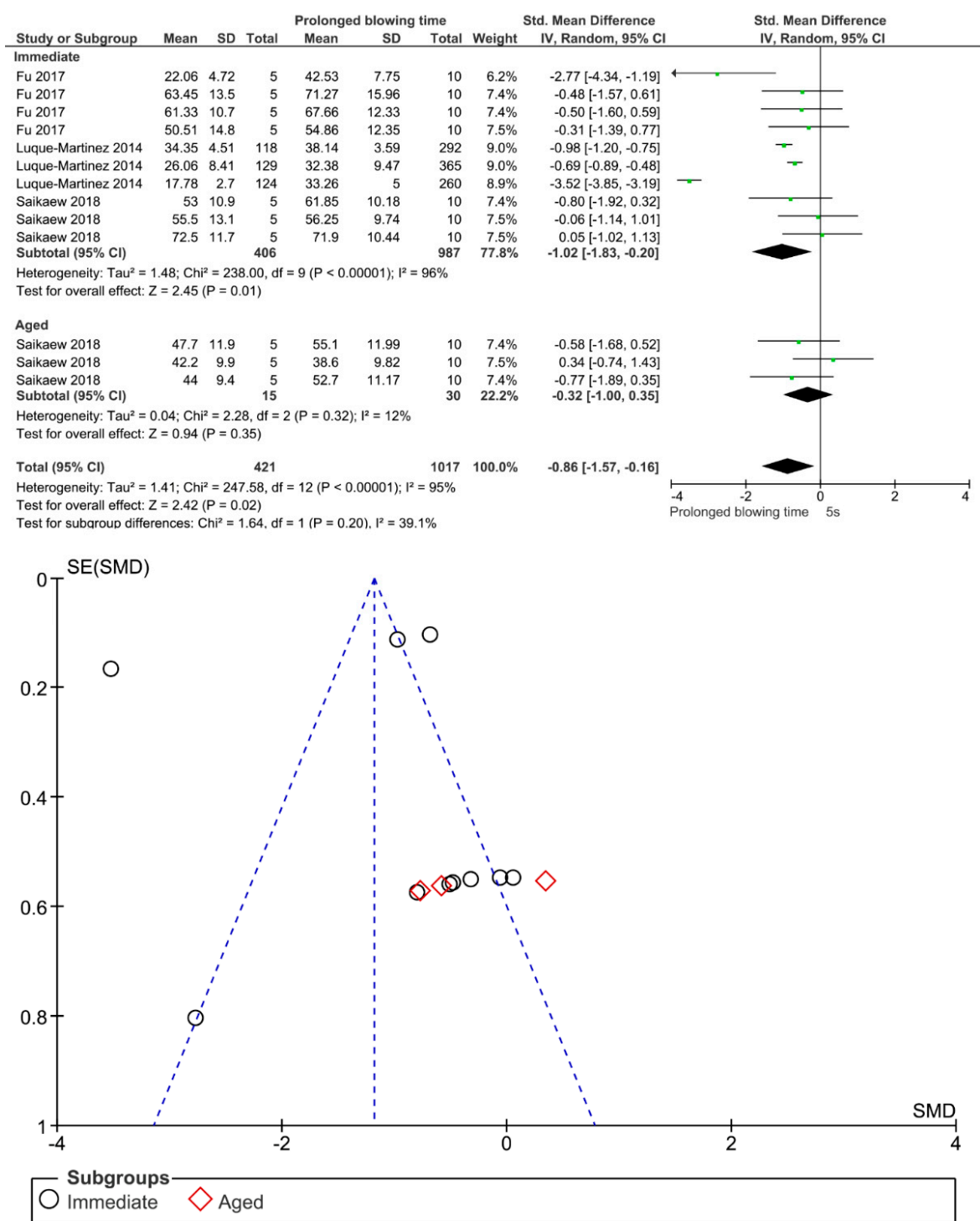

Figure 12. Results of the meta-analysis of immediate and aged bond strength of universal adhesive to dentin with prolonged solvent evaporation time. 


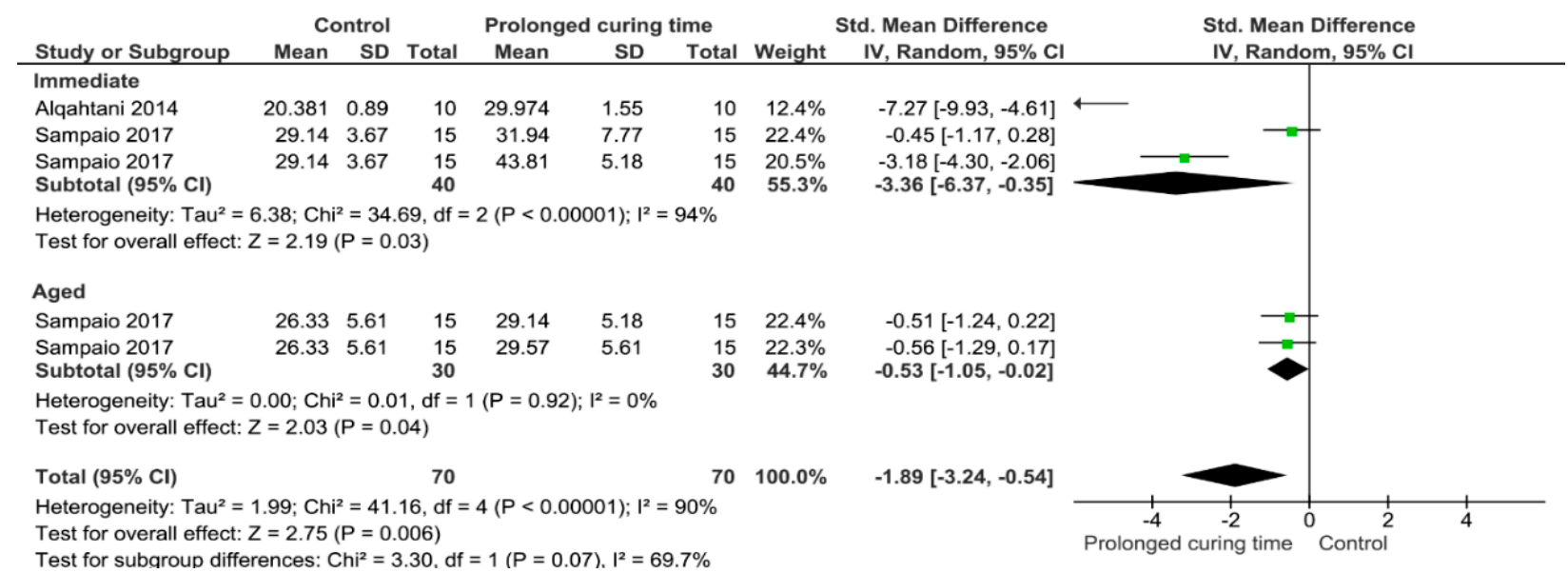

Figure 13. Results of the meta-analysis of immediate and aged bond strength of universal adhesive to dentin with prolonged curing time.

\begin{tabular}{|c|c|c|c|c|c|c|c|c|c|c|c|}
\hline \multirow{2}{*}{ Study or Subgroup } & \multicolumn{3}{|c|}{ Control } & \multicolumn{3}{|c|}{ Scrubbing technique } & \multicolumn{2}{|r|}{ Std. Mean Difference } & \multirow{2}{*}{\multicolumn{3}{|c|}{$\begin{array}{l}\text { Std. Mean Difference } \\
\text { IV, Random, } 95 \% \mathrm{CI}\end{array}$}} \\
\hline & Mean & SD & Total & Mean & SD & Total & Weight & IV, Random, 95\% Cl & & & \\
\hline \multicolumn{12}{|l|}{ Immediate } \\
\hline Irmak 2018 & 21.23 & 5.81 & 6 & 31.1 & 8.17 & 12 & $6.5 \%$ & $-1.25[-2.33,-0.17]$ & & & \\
\hline Irmak 2018 & 25.15 & 5.05 & 6 & 26.8 & 6.17 & 12 & $7.8 \%$ & $-0.27[-1.25,0.72]$ & & & \\
\hline Jang 2018 & 23.5 & 4.97 & 30 & 27.9 & 5.71 & 30 & $27.3 \%$ & $-0.81[-1.34,-0.28]$ & & & \\
\hline $\begin{array}{l}\text { Thanatvarakorn } 2016 \\
\text { Subtotal }(95 \% \mathrm{Cl})\end{array}$ & 57.25 & 9.29 & $\begin{array}{l}20 \\
62\end{array}$ & 66.26 & 10.1 & $\begin{array}{l}20 \\
74\end{array}$ & $\begin{array}{l}17.8 \% \\
59.4 \%\end{array}$ & $\begin{array}{l}-0.91[-1.56,-0.26] \\
-0.82[-1.18,-0.46]\end{array}$ & & & \\
\hline \multicolumn{12}{|c|}{$\begin{array}{l}\text { Heterogeneity: } \mathrm{Tau}^{2}=0.00 ; \mathrm{Chi}^{2}=1.88, \mathrm{df}=3(\mathrm{P}=0.60) ; \mathrm{I}^{2}=0 \% \\
\text { Test for overall effect: } Z=4.48(\mathrm{P}<0.00001)\end{array}$} \\
\hline \multicolumn{12}{|l|}{ Aged } \\
\hline Irmak 2018 & 26.05 & 4.16 & 6 & 30.58 & 7 & 12 & $7.4 \%$ & $-0.69[-1.70,0.32]$ & & - & \\
\hline Irmak 2018 & 22.41 & 5.76 & 6 & 34.81 & 6.93 & 12 & $5.4 \%$ & $-1.79[-2.98,-0.61]$ & & & \\
\hline $\begin{array}{l}\text { Jang } 2018 \\
\text { Subtotal }(95 \% \mathrm{CI})\end{array}$ & 22.3 & 3.16 & $\begin{array}{l}30 \\
42\end{array}$ & 25.4 & 5.05 & $\begin{array}{l}30 \\
54\end{array}$ & $\begin{array}{l}27.8 \% \\
40.6 \%\end{array}$ & $\begin{array}{l}-0.73[-1.25,-0.20] \\
-0.92[-1.49,-0.35]\end{array}$ & & & \\
\hline \multicolumn{12}{|c|}{$\begin{array}{l}\text { Heterogeneity: } \mathrm{Tau}^{2}=0.08 ; \mathrm{Ch}^{2}=2.75, \mathrm{df}=2(P=0.25) ; \mathrm{I}^{2}=27 \% \\
\text { Test for overall effect: } Z=3.18(P=0.001)\end{array}$} \\
\hline Total $(95 \% \mathrm{Cl})$ & & & 104 & & & 128 & $100.0 \%$ & $-0.84[-1.11,-0.56]$ & & & \\
\hline $\begin{array}{l}\text { Heterogeneity: } \mathrm{Tau}^{2}= \\
\text { Test for overall effect: } \\
\text { Test for subaroup differ }\end{array}$ & $\begin{array}{l}00 ; \mathrm{Chi}^{2} \\
=5.94(\mathrm{~F} \\
\text { ences: } \mathrm{Cr}\end{array}$ & $\begin{array}{l}=4.66 \\
P<0.0 \\
h i^{2}=0\end{array}$ & $\begin{array}{l}\text {, df }=6 \\
0001) \\
09, d f=\end{array}$ & $P=0.5$ & $1^{2}=0 \%$ & & & & $-4 \quad-2$ & Control & 2 \\
\hline
\end{tabular}

Figure 14. Results of the meta-analysis of immediate and aged bond strength of universal adhesive to dentin applied in an activated mode.

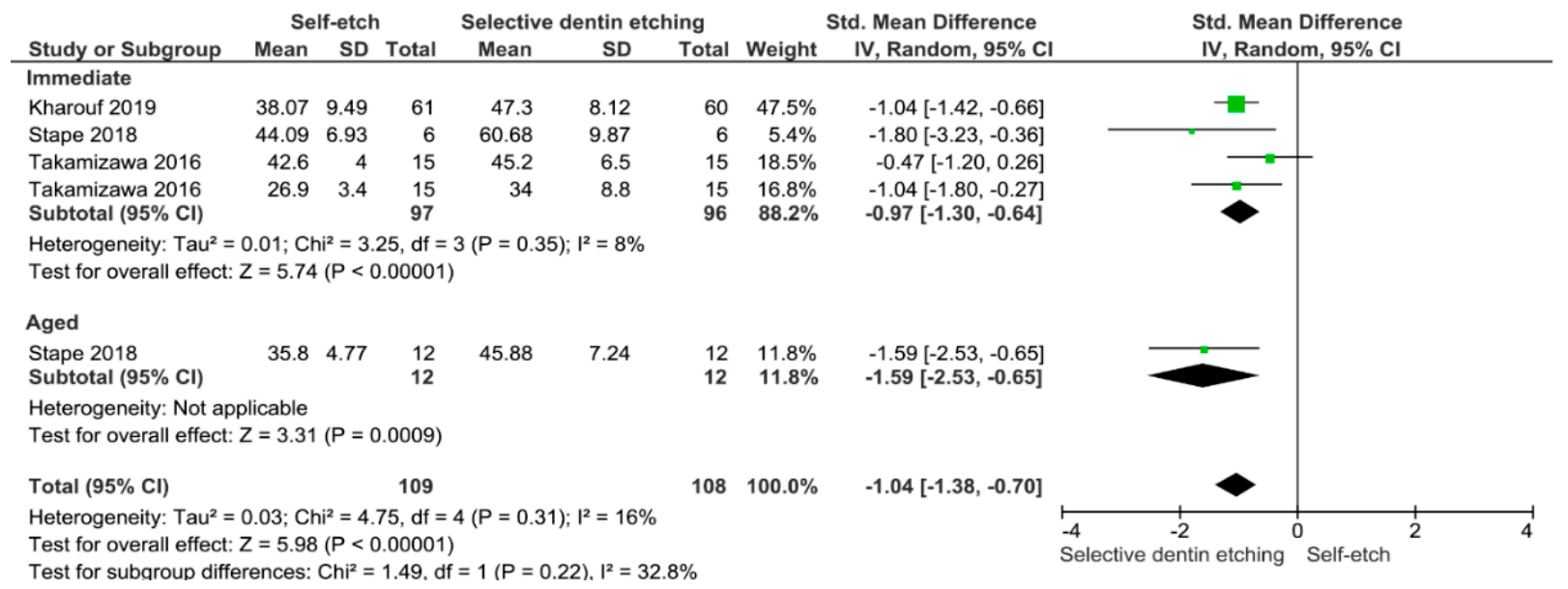

Figure 15. Results of the meta-analysis of immediate and aged bond strength of universal adhesive to dentin using the selective dentin etching. 


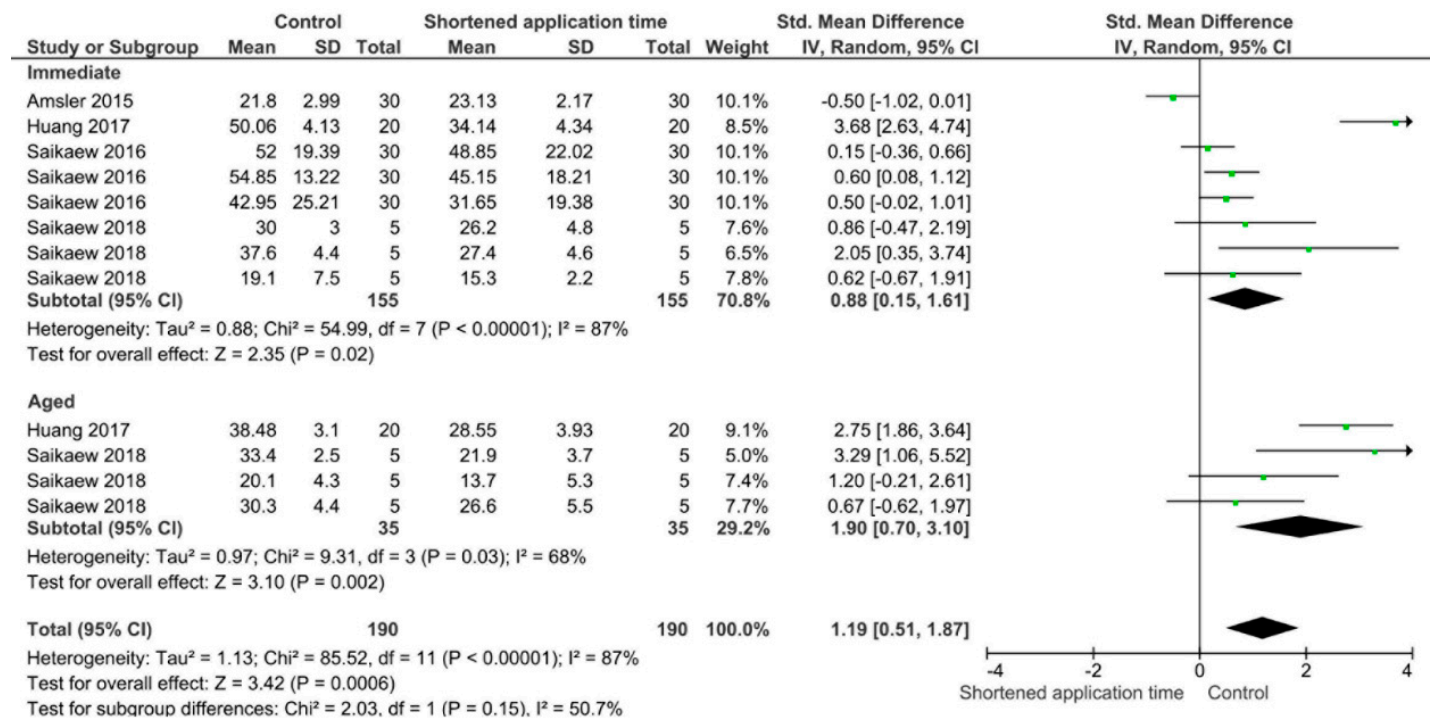

Test for subaroup differences: $\mathrm{Ch}^{2}=2.03, \mathrm{df}=1(\mathrm{P}=0.15), \mathrm{I}^{2}=50.7 \%$

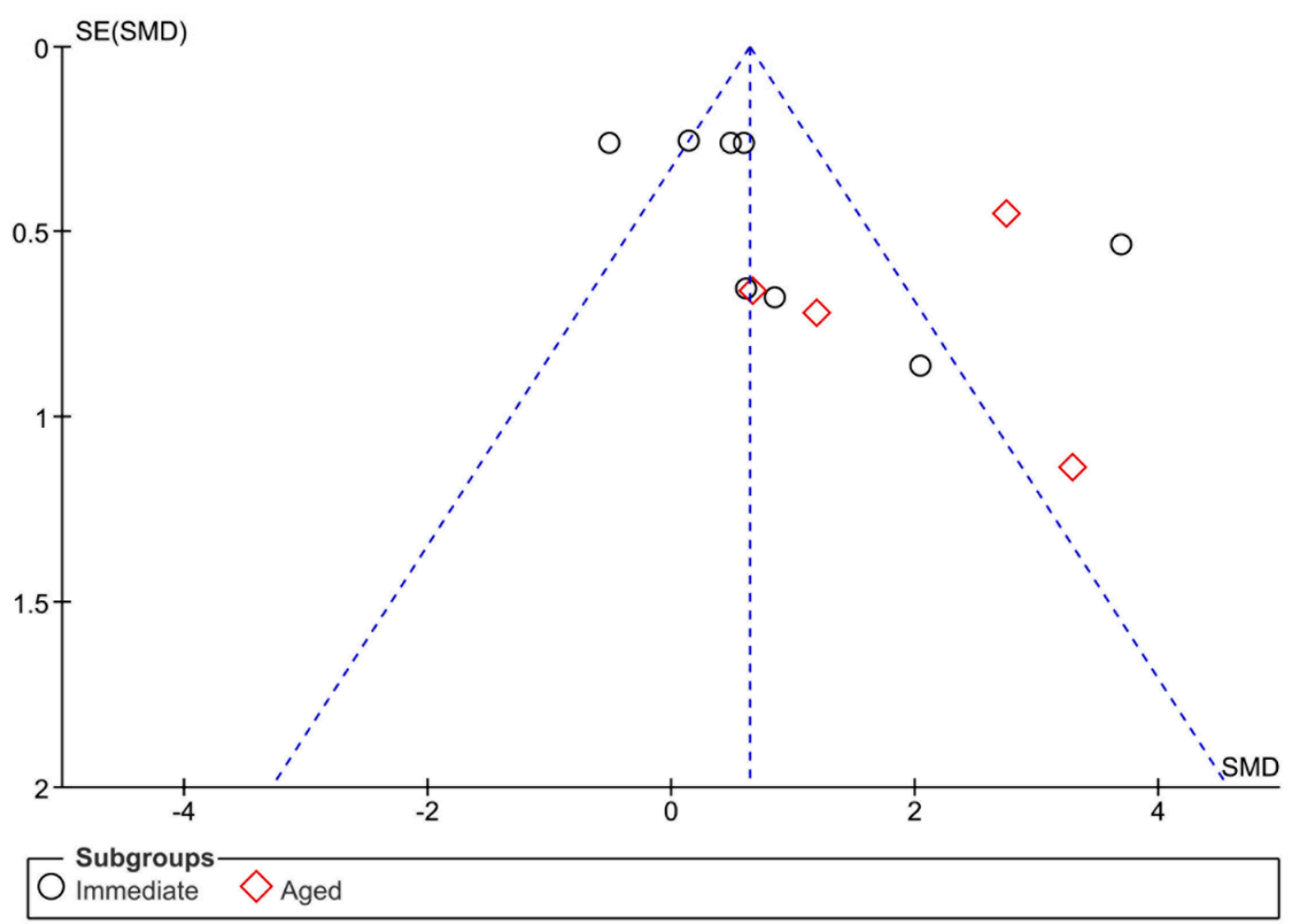

Figure 16. Results of the meta-analysis of immediate and aged bond strength of universal adhesive to dentin applied using a shortened application time.

On the other hand, the use of a shortened application time $(p<0.001)$ and dentin desensitizers $(p=0.01)$ impaired the bond strength of universal adhesives to dentin. Finally, the use of lasers $(p=0.06)$, air abrasion $(p=0.016)$, and crosslinking agents $(p=0.08)$ prior to the application of a universal adhesive did not have any effect on the bond strength.

According to the parameters for methodological quality assessment, most studies included were classified with medium risk of bias (Figure 17). Most of the studies analyzed failed to report the single operator, operator blinded, and sample size calculation parameters. 

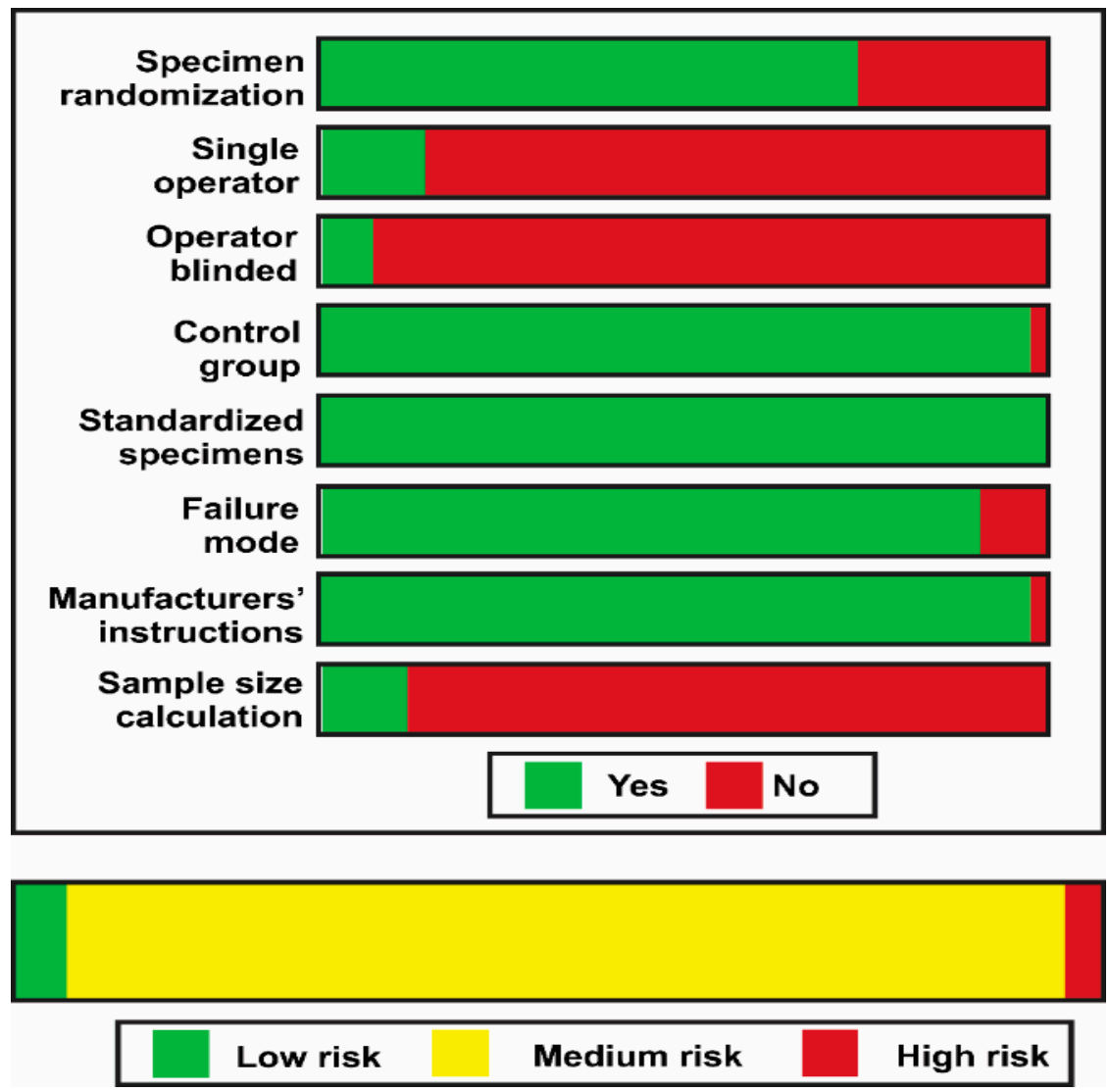

Figure 17. Summary of the results of the risk of bias assessment.

\section{Discussion}

A systematic review and meta-analysis was conducted regarding the bond strength of universal adhesives to dentin depending on the additional strategy used: Extra hydrophobic coat, prolonged curing time, multiple layer application, prolonged blowing time, dentin desensitizer, air abrasion, ethanol-wet bonding technique, non-thermal atmospheric plasma, selective dentin etching for $3 \mathrm{~s}$, scrubbing technique, laser technology, shortened application time, prolonged application time, MMP inhibitors, and crosslinking agents. Some of the techniques mentioned above improved the bond strength of the universal adhesives to dentin, while others lacked any effect, and many others impaired the bond strength. Therefore, the hypothesis tested in this study was partially accepted.

For an extra hydrophobic coat application, both immediate and aged bond strength were improved. One should bear in mind that the bond durability of universal adhesives to dentin might be limited by both their thinnest film thickness and thin hybrid layer [117]. Nevertheless, the clinical benefit of a thicker adhesive layer is still scant in literature. In this regard, the placement of an extra hydrophobic coat has been undoubtedly used recently to improve the bonding performance of universal adhesives to dentin $[118,119]$, by means of a higher hydrophobicity, and superior polymerization [79,80]. Moreover, better sealing of the adhesive interface, which would indicate better bond degradation prevention, could be possible by the additional of a hydrophobic layer [120]. The aforementioned statement could be explained by the phenomena in which the fluid flow across the adhesive interface decreases due to the increase in the adhesive layer thickness $[81,121]$. Numerous studies advocated the fact that the short and long-term bonding performance of one-step-self-etch adhesive systems could be improved by placement of such an extra layer [120,122]. In this respect, aging stability could be material dependent [79], and the use of an additional hydrophobic coat could be more beneficial for self-etch rather than the etch-and-rinse strategy [81]. Overall, the meta-analysis showed that the extra layer of 
hydrophobic coat supplements unsolvated monomers with hydrophobic nature into adhesive interface, which subsequently reduces the concentration of unreacted monomers and retained solvents in the adhesive layer [123], increasing the bond strength of the adhesive interface [124].

Immediate and aged bond strength were increased by increasing the photoactivation time. Ideally, enough energy must be applied by a restorative material to convert monomers into stable polymers [103]. To improve polymerization and reduce permeability, increasing the time of light exposure of adhesives beyond the manufacturer's recommendation has been suggested [86]. This technique has been proved to guarantee an adequate degree of conversion, [110] making the adhesive less prone to water sorption [125]. In addition, extended curing time could maintain the camphorquinone in a state which allows it to react with a co-initiator, producing a higher energy density and increasing free radical formation [126]. In this manner, the exposure time recommended by the respective manufacturer for adhesive systems is not sufficient to obtain an optimal polymerization [125,127]. Hence, a longer exposure time up to $40 \mathrm{~s}$ is preferred, as was demonstrated by this meta-analysis.

Immediate bond strength is increased by using multiple layer application; however, this effect could be not observed after aging. Previous studies suggested that doubling the number of adhesive layers improves bond strength by enhancing monomer penetration into hybrid layers and increasing chemical interactions $[93,128]$. Therefore, an additional layer application should be considered as a crucial clinical step. Almost all universal adhesives were based on the functional monomer 10-MDP (methacryloyloxydecyl dihydrogen phosphate) imperative to obtain stable nanolayer structure (10-MDP/Ca salts) in the hybrid layer and adhesive layer [11]. MDP monomer needs an appropriate time of $20 \mathrm{~s}$ for its chemical interaction to take place; however, applying a second coat of such a monomer without curing the first one permits the first layer to sufficiently interact with hydroxyapatite and thus promotes supplementary bonding [129].

Immediate bond strength was improved by prolonged blowing time; however, this effect could be not observed after aging. In the bonding procedure, air-drying for solvent evaporation is considered a crucial step after the application of the adhesive. Since ethanol and water can form hydrogen bonds with each other and also with the monomer, it is difficult to remove water from ethanol-based adhesives compared to those of acetone-based adhesives [130]. In a study by Saikaew et al., longer air-blowing time caused significant increases in the bond strength of ethanol-based adhesives, whereas the bond strength of acetone-based adhesive was not affected by the different air-blowing time. This conclusion seems to partially support the results in this meta-analysis, as improvement in bond strength was not observed in all adhesives after aging. In this sense, prolonged air-blowing time for 15-30 s could be suggested in order to enhance immediate adhesive properties of some universal adhesives [113].

The use of dentin desensitizer impaired both immediate and aged bond strength. The application of dentin desensitizers aids in occluding dentinal tubules and its combination with adhesive systems could alleviate the post-operative sensitivity accompanying the placement of composite resin [131]. However, its influence on bonding performance by means of diverse bonding agents remains to be assessed as the desensitized treated dentin might be harmful for bonding [132]. This could be explained by the formation of nanoparticle layers distributed inside dentin substrates which impede the interaction of adhesive to demineralized dentin [133]. Additionally, resin tags formation might be disturbed by tubule occlusion with calcium-containing desensitizer pastes, resulting in diminished bond strength [134].

The use of air abrasion did not have any significant effect on both immediate and aged bond strength. Despite this, it's important to note that by modifying dentin with airborne particles may negatively affect the chemical interaction between functional monomers and hydroxyapatite. This could be explained by the remnant particles resulting from the air abrasion application [29]. Dentin might experience damage by harder particles like alumina particles as a result of its lower elastic modulus and lower hardness [135]. Furthermore, 
these particles are able to chemically and/or mechanically alter the collagen fibers, which hinders the adhesive penetration and consequently lessens the bond strength [32].

Immediate bond strength was improved by the use of ethanol-wet bonding technique. Ethanol is acknowledged to be a solvent of choice when compared to water since it is able to reduce the diameter of collagen fibrils and increase the interfibrillar space, allowing monomers to easily infiltrate the collagen fibrils [136]. This technique, called "ethanol-wet bonding technique", has been proven to efficiently seal the dentinal matrix, reducing the dentin-resin interface permeability, which would reduce the activity of collagenolytic enzymes and consequently improve bond durability to dentin substrate [137]. In this simplified technique, $100 \%$ of ethanol is applied for $1 \mathrm{~min}$, which could be clinically acceptable [138], when collagens are successfully protected with ethanol, greater longevity of adhesive interface is observed, creating a stable bond [138].

The use of non-thermal atmospheric plasma improves the aged bond strength, but presents no effect on the immediate bond strength. Plasma has been reported to improve bonding to dentin [139]. It is described as partially ionized gases that comprise electronically excited atoms, molecules, ions, and free radical species [100]. These particles are considered as reactive crosslinkers, able to form chemical functional groups on the surface of dentin [139]. The use of non-thermal atmospheric plasma proved to increase dentin wettability $[140,141]$, improve resin polymerization [142], and enhance resin infiltration $[143,144]$. Another potential explanation for the bond-promotion influence is that non-thermal atmospheric plasma stimulates the dentin substrate by leaving free radicals or peroxides, thus strengthening the interaction between collagen fibrils and resin monomers [145]. Furthermore, a reduction in the expression and activity of MMP-2 and MMP-9 could be possible by applying a non-atmospheric plasma on thyroid papillary cancer cells [103].

Both immediate and aged bond strength were improved by the application of selective dentin etching for $3 \mathrm{~s}$. Van Meerbeek et al. suggested that protecting collagen from hydrolysis and early degradation of the bond could be achieved by keeping hydroxyapatite around collagen fibrils [146]. Therefore, a functional monomer such as (10-MDP), which is found inside universal adhesives, can form stable calcium-phosphate complexes and self-assemble into the form of a regular layered structure at the apatite surface. Maintaining calcium at the bonded interface may favor this uncharacteristic chemical bonding process. Consequently, in order to improve the resin-dentin bonding, a new approach called "Selective dentin etching" has emerged. This technique is based on the application of phosphoric acid during $3 \mathrm{~s}$ in dentin, which after rinsing and drying, leaves a partially demineralized substrate [110-112]. In general, the remaining calcium-content ratio was dependent on the time of etching; the following residual calcium-content ratios were found to be similar on self-etch mode and $3 \mathrm{~s}$ of selectively etched dentin. Etching times above $5 \mathrm{~s}$ considerably lessen these ratios [111]. This finding clearly indicates that this technique has been proven as an alternative to improve the long-term bond strength of universal adhesives to dentin.

The use of scrubbing technique improves both immediate and aged bond strength. Active application of universal adhesives enhanced dentin bond durability of the etching mode by facilitating the penetration of adhesives into the branches of dentin tubules, and improving solvent evaporation [109]. Actually, the active application of adhesives using a scrubbing technique leads to the impregnation of a higher rate of monomers inside the smear layer, hence improving adhesive-interface quality and decreasing hybrid layer degradation $[147,148]$. After a manual force is applied through an adhesive rubbing motion into the dentin substrate, dentin acts similar to a sponge, and then the dentin collagen network is compressed. Compressed collagen enlarges as the pressure is relieved and the infiltration of the adhesive monomers into collagen network is enhanced [149]. Furthermore, since monomers like 10-MDP with hydrophobic nature are well impregnated due to adhesive agitation, aging couldn't negatively affect bonding performance [37,150].

Immediate bond strength was impaired by the use of lasers, while aged bond strength showed no statistical difference. Overall, there was no difference between control and 
laser. Laser technology was initiated in the 20th century, to be an alternative technique to the conventional drilling technique [151]. Lasers had the ability to absorb water and hydroxyapatite necessary for hard tissue removal [152]. Higher absorption during laser ablation could be the reason for carious removal by means of micro-explosion, water vaporization, and heating [153]. A clear substrate, free of debris, could be achieved by such a laser, but considering adhesion to dentin, numerous studies revealed a decrease in bond strength with the use of Er:YAG laser [154,155]. This could be explained by the fact that when applying a laser, water and organic constituents of dentin vaporize, producing the thermomechanical ablation of the inorganic constituents [156]. Collagen networks fuse by dentinal ablation, causing a lack of interfibrillar space, and accordingly, resin monomers will not be able to penetrate adequately, affecting the bond strength negatively [157].

The choice between shortened application time and prolonged application time may direct dentists toward the shortened application time. However, this study's results showed that the shortened application time impairs both immediate and aged bond strength Manufacturers of universal adhesives simplify the application technique, nevertheless, Saikaew et al. (2016) revealed certain weaknesses with reduced application time on immediate dentin bond strength [116]. This could be in agreement with the finding of this study and could be explained by the fact that three factors affected the reduced application time, these factors being: Smear layer interaction, method of application, and adhesive composition. These factors could be considered for each adhesive [106]. Acetone had a high vapor pressure compared to water and ethanol, so a longer application time for acetone-based adhesive could enhance the evaporation of such a solvent and increase bond strength; however, this is not the case with ethanol-based adhesives, since water inside universal adhesives forms a hydrogen bond with ethanol and with the shortened application time, the ethanol evaporation might be affected and the bond strength stability diminishes [130]. Accordingly, the residual water and solvent might compromise the polymerization of adhesive, which could accelerate monomer degradation with time and impair bond strength [158]. This statement is in agreement with this meta-analysis. With regards to smear layer interaction, shortened application time may not dissolve the smear layer easily, and the bond strength could be compromised [106,115]. The longer the adhesive-smear layer interaction, the better the penetration of monomers through the smear layer to form a stronger bond with the dentin substrate $[106,159]$.

Prolonged application time improves immediate bond strength, while aged bond strength showed no statistical difference. With longer application time of adhesives, a better performance of resin-dentin bonds could be achieved, and monomers are able to diffuse inward [128]. This is responsible for increasing the saturation of collagen by resin since monomers ideally should fill the space between the exposed collagen fibrils $[160,161]$, otherwise adhesive durability could be affected. In addition, when the adhesive is applied for a prolonged period of time, more solvent can evaporate, hence permitting the development of a stronger polymer within the dentin substrate and higher resin-dentin bond strength [96,162]. Therefore, increasing the application time enhances the chemical reaction between functional monomers and hydroxyapatite, which is considered a crucial step for increasing monomer infiltration [163].

MMP inhibitors improve immediate and long-term bond strength of universal adhesive to dentin. Regardless of the adhesive system used, a major problem is the degradation of the hybrid layer over time. It is well known that the dental adhesion process is based on the creation of a suitable and compact hybrid layer created by impregnating the dentin substrate with monomers, rather on the morphology or the thickness of the hybrid layer [164], in general, the more compact and homogeneous the hybrid layer, the better the stability of the resin-dentin bond [165]. When a portion of collagen remained unprotected within the hybrid layer, a cleavage by endogenous and exogenous collagenolytic enzymes of the family endopeptidases, like the matrix metalloproteinases (MMPs), can be assumed [7]. In order to improve the quality of the resin-dentin interface, some studies focused on counteracting enzymatic biodegradation by the use of matrix metalloproteinases (MMPs) inhibitors [166]. 
The use of MMP inhibitors on dentin substrate had been advocated to improve the longevity of adhesive and to reduce aging over time by means of altering the active site of MMPs [123]. These inhibitors include chlorhexidine, 1-ethyl-3-(3-dimethylaminopropyl) carbodiimide, green tea extract, galardin, tetracyclines and analogues, quaternary ammonium salts, and ethylenediaminetetraacetic acid [167-170]. This conclusion seems to support the finding of this study, as the use of MMP inhibitors lessens interfacial aging over time by hindering the activation of endogenous dentin enzymes, which are dependable for the degradation of collagen network.

Immediate bond strength was not improved by the use of crosslinking agents, while aged bond strength showed an improvement. Overall, there was no difference between control and crosslinking agents. Since higher mechanical properties and lower biodegradation rates of collagen are needed, the use of collagen crosslinking agents in adhesive procedures have gained interest [5]. Covalent cross-links created with external cross-linkers such as ribose, glutaraldehyde, carbodiimide hydrochloride, proanthocyanidins, chlorhexidine, riboflavin, chitosan, polyphenols, and genipin have been found to be very stable, by offering significant advantages in developing mechanically stable collagen scaffolds [171]. Ideally, the introduction of additional cross-links prevents collagen molecules inside dentin from sliding past each other under stress [172], which increases the mechanical strength of collagen fibers and reduces extensibility [47]. Collagen crosslinking was initiated as a substitute dentin pre-treatment to strengthen dentinal collagen fibrils, as well as enhance the bonding durability [173,174]. This matches with the finding of this manuscript, as the effect of crosslinkers appear with time to reinforce collagen by means of maintenance of an expanded position which is able to receive solvents and monomers $[175,176]$.

The methodological quality assessment revealed that most studies included were classified with medium risk of bias, which indicate that the quality of the evidence of the outcome evaluated could be high. In relation to this, it should be highlighted that blinding and sample size calculation were not reported in most of the studies analyzed, and failure to describe these parameters could increase the likelihood of performance, attrition, and detection bias [177].

From this review, in vitro evidence was evaluated with regards to the alternative techniques used in the literature for the bonding efficacy of universal adhesives to dentin substrate. The results of this review should be considered with caution since, in clinical situations, a wet environment, masticatory stresses, and $\mathrm{pH}$, lead to a rapid adhesive-dentin interface. Future research must be conducted, especially randomized controlled clinical trials, with the purpose of gaining a better understanding of the performance of universal adhesives in the clinical success of resin-based restorations to dentin substrate.

\section{Conclusions}

With the limitation of a long distance between laboratory studies and clinical randomized evaluations, the in vitro evidence suggests that the application of universal adhesives using some alternative techniques or additional strategies different than the manufacturers' recommendations may be beneficial for improving the bonding performance to dentin. Due to its practicality, the active application of the adhesive, together with the evaporation of the solvent for periods of time longer than $10 \mathrm{~s}$, could be recommended for improving the bond strength values to dentin. In addition, prolonged curing time of up to $40 \mathrm{~s}$, selective dentin etching for $3 \mathrm{~s}$, double adhesive application time, extra hydrophobic resin layer, previous application of MMP inhibitors, non-thermal atmospheric plasma, ethanol-wet bonding strategy, and multiple layer application (2 layers and more) are recommended. On the other hand, shortened application time, and application of desensitizer to the dentin should be avoided since the bond strength could be impaired. 
Supplementary Materials: The following are available online at https:/ / www.mdpi.com/2073-436 0/13/5/814/s1, Table S1: Search strategy used in ISI Web of Science; Table S2: Search strategy used in SCOPUS; Table S3: Search strategy used in EMBASE; Table S4: Excluded studies.

Author Contributions: Conceptualization, L.H., R.B., and C.E.C.-S.; methodology, L.H., R.B., and C.E.C.-S.; software, L.H., R.B., and C.E.C.-S.; validation, L.H., R.B., C.E.C.-S., and N.K.; formal analysis, R.B. and C.E.C.-S.; investigation, L.H., R.B., C.E.C.-S. and N.K.; resources, L.H., C.E.C.-S., D.M., Y.H., and R.B.; data curation, L.H., R.B., and C.E.C.-S.; writing-original draft preparation, L.H., R.B., and C.E.C.-S.; writing-review and editing, L.H., M.Z., Y.H., N.K., N.J., C.E.C.-S., and R.B.; visualization, L.H., R.B., N.K., M.Z., N.J., D.M., and C.E.C.-S.; supervision, L.H.; project administration, L.H. and C.E.C.-S. All authors have read and agreed to the published version of the manuscript.

Funding: This research received no external funding.

Data Availability Statement: The data that support the findings of this study are available from the corresponding author upon reasonable request.

Acknowledgments: Authors Louis Hardan and Rim Bourgi would like to acknowledge the SaintJoseph University of Beirut, Lebanon. The authors would also thank the University of Hidalgo State, Mexico and the University of Strasbourg for supporting this research.

Conflicts of Interest: The authors declare no conflict of interest.

\section{References}

1. Van Meerbeek, B.; Peumans, M.; Poitevin, A.; Mine, A.; Van Ende, A.; Neves, A.; De Munck, J. Relationship between bond-strength tests and clinical outcomes. Dent. Mater. 2010, 26, e100-21. [CrossRef] [PubMed]

2. Spencer, P.; Wang, Y.; Katz, J.L. Identification of collagen encapsulation at the dentin/adhesive interface. J. Adhes. Dent. 2004, 6, 91-95. [PubMed]

3. Stape, T.H.S.; Tjäderhane, L.; Abuna, G.; Sinhoreti, M.A.C.; Martins, L.R.M.; Tezvergil-Mutluay, A. Optimization of the etch-andrinse technique: New perspectives to improve resin-dentin bonding and hybrid layer integrity by reducing residual water using dimethyl sulfoxide pretreatments. Dent. Mater. 2018, 34, 967-977. [CrossRef] [PubMed]

4. Lima, J.M.C.; Anami, L.C.; Pereira, S.M.B.; de Melo, R.M.; Bottino, M.A.; de Miranda, L.M.; Souza, K.B.; Özcan, M.; Souza, R.O.A. Dentin/composite bond strength: Effect of aging and experimental unit. J. Adhes. Sci. Technol. 2020, 1-11. [CrossRef]

5. Frassetto, A.; Breschi, L.; Turco, G.; Marchesi, G.; Di Lenarda, R.; Tay, F.R.; Pashley, D.H.; Cadenaro, M. Mechanisms of degradation of the hybrid layer in adhesive dentistry and therapeutic agents to improve bond durability-A literature review. Dent. Mater. 2016, 32, e41-e53. [CrossRef]

6. Cardoso, S.A.; Oliveira, H.L.; Münchow, E.A.; Carreño, N.L.; Gonini Junior, A.; Piva, E. Effect of shelf-life simulation on the bond strength of self-etch adhesive systems to dentin. Appl. Adhes. Sci. 2015, 3. [CrossRef]

7. Betancourt, D.E.; Baldion, P.A.; Castellanos, J.E. Resin-Dentin Bonding Interface: Mechanisms of Degradation and Strategies for Stabilization of the Hybrid Layer. Int. J. Biomater. 2019, 2019, 5268342. [CrossRef] [PubMed]

8. Takamizawa, T.; Imai, A.; Hirokane, E.; Tsujimoto, A.; Barkmeier, W.W.; Erickson, R.L.; Latta, M.A.; Miyazaki, M. SEM observation of novel characteristic of the dentin bond interfaces of universal adhesives. Dent. Mater. 2019, 35, 1791-1804. [CrossRef] [PubMed]

9. Masarwa, N.; Mohamed, A.; Abou-Rabii, I.; Abu Zaghlan, R.; Steier, L. Longevity of Self-etch Dentin Bonding Adhesives Compared to Etch-and-rinse Dentin Bonding Adhesives: A Systematic Review. J. Evid. Based Dent. Pract. 2016, 16, 96-106. [CrossRef] [PubMed]

10. Geng Vivanco, R.; Cardoso, R.S.; Sousa, A.B.S.; Chinelatti, M.A.; de Vincenti, S.A.F.; Tonani-Torrieri, R.; Pires-de-Souza, F.D.C.P. Effect of thermo-mechanical cycling and chlorhexidine on the bond strength of universal adhesive system to dentin. Heliyon 2020, 6, e03871. [CrossRef]

11. Papadogiannis, D.; Dimitriadi, M.; Zafiropoulou, M.; Gaintantzopoulou, M.-D.; Eliades, G. Universal Adhesives: Setting Characteristics and Reactivity with Dentin. Materials 2019, 12, 1720. [CrossRef] [PubMed]

12. Cuevas-Suárez, C.E.; de Oliveira da Rosa, W.L.; Vitti, R.P.; da Silva, A.F.; Piva, E. Bonding Strength of Universal Adhesives to Indirect Substrates: A Meta-Analysis of in Vitro Studies. J. Prosthodont. 2020, 29, 298-308. [CrossRef]

13. Cuevas-Suárez, C.E.; De Oliveira da Rosa, W.L.; Lund, R.G.; da Silva, A.F.; Piva, E. Bonding Performance of Universal Adhesives: An Updated Systematic Review and Meta-Analysis. J. Adhes. Dent. 2019, 21, 7-26. [CrossRef] [PubMed]

14. Van Meerbeek, B.; Yoshihara, K.; Yoshida, Y.; Mine, A.J.; De Munck, J.; Van Landuyt, K.L. State of the art of self-etch adhesives. Dent. Mater. 2011, 27, 17-28. [CrossRef]

15. Nagarkar, S.; Theis-Mahon, N.; Perdigão, J. Universal dental adhesives: Current status, laboratory testing, and clinical performance. J. Biomed. Mater. Res. 2019, 107, 2121-2131. [CrossRef] [PubMed] 
16. Bourgi, R.; Hardan, L.; Rivera-Gonzaga, A.; Cuevas-Suárez, C.E. Effect of warm-air stream for solvent evaporation on bond strength of adhesive systems: A systematic review and meta-analysis of in vitro studies. Int. J. Adhes. Adhes. 2021, 105, 102794. [CrossRef]

17. Page, M.; McKenzie, J.; Bossuyt, P.; Boutron, I.; Hoffman, T.; Mulrow, C.; Shamseer, L.; Tetzlaff, J.; Akl, E.; Brennan, S.; et al. The PRISMA 2020 statement: An Updated Guideline for Reporting Systematic Reviews. MetaArXiv Prepr. 2020. Available online: https:/ / osf.io/preprints/metaarxiv/v7gm2/ (accessed on 2 March 2021).

18. Jones, P.M. COMBINE: Stata Module to Combine n, Mean, and SD from Two Groups According to the CochraneRECOMMENDED Formula for Meta-Analyses. 2011. Available online: https://ideas.repec.org/c/boc/bocode/s457265.html (accessed on 2 March 2021).

19. Anja, B.; Walter, D.D.; Nicoletta, C.; Marco, F.; Pezelj Ribaric, S.; Ivana, M.M.; Pezelj Ribarić, S.; Ivana, M.M. Influence of Air Abrasion and Sonic Technique on Microtensile Bond Strength of One-Step Self-Etch Adhesive on Human Dentin. Sci. World J. 2015, 2015, 368745. [CrossRef]

20. Alaghehmand, H.; Nezhad Nasrollah, F.; Nokhbatolfoghahaei, H.; Fekrazad, R. An In Vitro Comparison of the Bond Strength of Composite to Superficial and Deep Dentin, Treated With Er:YAG Laser Irradiation or Acid-Etching. J. Lasers Med. Sci. 2016, 7, 167-171. [CrossRef] [PubMed]

21. Martini, E.C.; Parreiras, S.O.; Gutierrez, M.F.; Loguercio, A.D.; Reis, A. Effect of different protocols in preconditioning with EDTA in sclerotic dentin and enamel before universal adhesives applied in self-etch mode. Oper. Dent. 2017. [CrossRef]

22. Shahabi, S.; Chiniforush, N.; Bahramian, H.; Monzavi, A.; Baghalian, A.; Kharazifard, M.J. The effect of erbium family laser on tensile bond strength of composite to dentin in comparison with conventional method. Lasers Med. Sci. 2013, 28, 139-142. [CrossRef] [PubMed]

23. Sinjari, B.; Santilli, M.; D'Addazio, G.; Rexhepi, I.; Gigante, A.; Caputi, S.; Traini, T. Influence of dentine pre-treatment by sandblasting with aluminum oxide in adhesive restorations. An in vitro study. Materials 2020, 13, 3026. [CrossRef] [PubMed]

24. Yan, H.; Wang, S.; Han, L.; Peng, W.; Yi, L.; Guo, R.; Liu, S.; Yang, H.; Huang, C. Chlorhexidine-encapsulated mesoporous silica-modified dentin adhesive. J. Dent. 2018, 78, 83-90. [CrossRef] [PubMed]

25. Zumstein, K.; Peutzfeldt, A.; Lussi, A.; Flury, S. The Effect of SnCl 2 / AmF Pretreatment on Short- and Long-Term Bond Strength to Eroded Dentin. Biomed Res. Int. 2018, 2018, 3895356. [CrossRef] [PubMed]

26. De Matos, N.R.S.; Costa, A.R.; Valdrighi, H.C.; Correr, A.B.; Vedovello, S.A.; Santamaria, M.J.; Correr-Sobrinho, L.; de Matos, N.R.S.; Costa, A.R.; Valdrighi, H.C.; et al. Effect of acid etching, silane and thermal cycling on the bond strength of metallic brackets to ceramic. Braz. Dent. J. 2016, 27, 734-738. [CrossRef]

27. Santos, M.J.M.C.C.; Costa, M.D.; Rego, H.M.C.; Rubo, J.H.; Santos, G.C. Effect of surface treatments on the bond strength of self-etching adhesive agents to dentin. Gen. Dent. 2017, 65, e1-e6. [PubMed]

28. Tsujimoto, A.; Barkmeier, W.W.; Takamizawa, T.; Latta, M.A.; Miyazaki, M. Effect of oxygen inhibition in universal adhesives on dentin bond durability and interfacial characteristics. Am. J. Dent. 2017, 30, 71-76.

29. Ouchi, H.; Takamizawa, T.; Tsubota, K.; Tsujimoto, A.; Imai, A.; Barkmeier, W.; Latta, M.; Miyazaki, M. The Effects of Aluminablasting on Bond Durability Between Universal Adhesives and Tooth Substrate. Oper. Dent. 2020, 45, 196-208. [CrossRef]

30. Poggio, C.; Beltrami, R.; Colombo, M.; Chiesa, M.; Beltrami, R.; Colombo, M.; Chiesa, M.; Scribante, A. Influence of dentin pretreatment on bond strength of universal adhesives. Acta Biomater. Odontol. Scand. 2017, 3, 30-35. [CrossRef]

31. Cardenas, A.F.M.F.M.; Siqueira, F.S.F.S.F.; Bandeca, M.C.C.; Costa, S.O.O.; Lemos, M.V.S.V.S.; Feitora, V.P.P.; Reis, A.; Loguercio, A.D.D.; Gomes, J.C.C. Impact of $\mathrm{pH}$ and application time of meta-phosphoric acid on resin-enamel and resin-dentin bonding. J. Mech. Behav. Biomed. Mater. 2018, 78, 352-361. [CrossRef]

32. Tamura, Y.; Takamizawa, T.; Shimamura, Y.; Akiba, S.; Yabuki, C.; Imai, A.; Tsujimoto, A.; Kurokawa, H.; Miyazaki, M. Influence of air-powder polishing on bond strength and surface-free energy of universal adhesive systems. Dent. Mater. J. 2017, 36, 762-769. [CrossRef] [PubMed]

33. Dos Santos, R.A.; de Lima, E.A.; Montes, M.A.J.R.; Braz, R. Pre-treating dentin with chlorhexadine and CPP-ACP: Self-etching and universal adhesive systems. Acta Biomater. Odontol. Scand. 2016, 2, 79-85. [CrossRef]

34. Şişmanoğlu, S. Bond durability of contemporary universal adhesives: Effect of dentin treatments and aging. J. Adhes. Sci. Technol. 2019, 33, 2061-2070. [CrossRef]

35. De Souza, M.Y.; DI Nicoló, R.; Bresciani, E. Influence of ethanol-wet dentin, adhesive mode of application, and aging on bond strength of universal adhesive. Braz. Oral Res. 2018, 32, e102. [CrossRef] [PubMed]

36. Ayar, M.K. Comparative evaluation of immediate bond strength to enamel with one-step self-etch adhesives. Res. J. Pharm. Biol. Chem. Sci. 2016, 7, 2014-2019.

37. Moritake, N.; Takamizawa, T.; Ishii, R.; Tsujimoto, A.; Barkmeier, W.; Latta, M.; Miyazaki, M. Effect of Active Application on Bond Durability of Universal Adhesives. Oper. Dent. 2019, 44, 188-199. [CrossRef]

38. Saito, T.; Takamizawa, T.; Ishii, R.; Tsujimoto, A.; Hirokane, E.; Barkmeier, W.; Latta, M.; Miyazaki, M. Influence of Application Time on Dentin Bond Performance in Different Etching Modes of Universal Adhesives. Oper. Dent. 2020, 45, 183-195. [CrossRef]

39. Da Rosa, L.S.; Follak, A.C.; Lenzi, T.L.; De Rocha, R.O.; Soares, F.Z.M. Phosphoric Acid Containing Chlorhexidine Compromises Bonding of Universal Adhesive. J. Adhes. Dent. 2018, 20, 243-247. [CrossRef] [PubMed]

40. Tsujimoto, A.; Barkmeier, W.W.; Takamizawa, T.; Latta, M.A.; Miyazaki, M. Influence of the Oxygen-inhibited Layer on Bonding Performance of Dental Adhesive Systems: Surface Free Energy Perspectives. J. Adhes. Dent. 2016, 18, 51-58. [CrossRef] [PubMed] 
41. Chiang, Y.-S.; Chen, Y.-L.; Chuang, S.-F.; Wu, C.-M.; Wei, P.-J.; Han, C.-F.; Lin, J.-C.; Chang, H.-T. Riboflavin-ultraviolet-A-induced collagen cross-linking treatments in improving dentin bonding. Dent. Mater. 2013, 29, 682-692. [CrossRef]

42. de Alencar, N.A.; Fidalgo, T.K.; Cajazeira, M.R.; Maia, L.C. Influence of the number of adhesive layers on adhesive interface properties under cariogenic challenge using streptococcus mutans. J. Adhes. Dent. 2014, 16, 339-346. [CrossRef] [PubMed]

43. Al Deeb, L.; Bin-Shuwaish, M.S.; Abrar, E.; Naseem, M.; Al-Hamdan, R.S.; Maawadh, A.M.; Al Deeb, M.; Almohareb, T.; Al Ahdal, K.; Vohra, F.; et al. Efficacy of chlorhexidine, Er Cr YSGG laser and photodynamic therapy on the adhesive bond integrity of caries affected dentin. An in-vitro study. Photodiagnosis Photodyn. Ther. 2020, 31, 101875. [CrossRef]

44. Deng, S.; Chung, K.; Chan, D.; Spiekerman, C. Evaluation of Bond Strength and Microleakage of a Novel Metal-titanate Antibacterial Agent. Oper. Dent. 2016, 41, E48-E56. [CrossRef] [PubMed]

45. Devarajan, S.S.; Madhubala, M.M.; Rajkumar, K.; James, V.; Srinivasan, N.; Mahalaxmi, S.; Sathyakumar, S. Effect of polydopamine incorporated dentin adhesives on bond durability. J. Adhes. Sci. Technol. 2021, 35, 185-198. [CrossRef]

46. Feiz, A.; Badrian, H.; Goroohi, H.; Mojtahedi, N. The Effect of Synthetic Grape Seed Extract (GSE) on the Shear Bond Strength of composite resin to Dentin. J. Res. Med. Dent. Sci. 2017, 5, 65-70.

47. Gu, L.; Shan, T.; Ma, Y.; Tay, F.R.; Niu, L. Novel Biomedical Applications of Crosslinked Collagen. Trends Biotechnol. 2019, 37, 464-491. [CrossRef] [PubMed]

48. Guarda, M.B.; Di Nizo, P.T.; Abuna, G.F.; Catelan, A.; Sinhoreti, M.A.C.; Vitti, R.P. Effect of Electric Current-assisted Application of Adhesives on their Bond Strength and Quality. J. Adhes. Dent. 2020, 22, 393-398. [CrossRef]

49. Cecchin, D.; Farina, A.; Vidal, C.; Bedran-Russo, A. A Novel Enamel and Dentin Etching Protocol Using $\alpha$-hydroxy Glycolic Acid: Surface Property, Etching Pattern, and Bond Strength Studies. Oper. Dent. 2018, 43, 101-110. [CrossRef]

50. Cangul, S.; Erpacal, B.; Adiguzel, O.; Sagmak, S.; Unal, S.; Tekin, S. Does the Use of Ozone as a Cavity Disinfectant Affect the Bonding Strength of Antibacterial Bonding Agents? Ozone Sci. Eng. 2020, 42, 565-570. [CrossRef]

51. Demirel, G.; Baltacioğlu, I.H. The influence of different etching modes and etching time periods on micro-shear bond strength of multi-mode universal adhesives on dentin. J. Stomatol. 2019, 72, 118-128. [CrossRef]

52. Akarsu, S.; Aktu_-Karademir, S. In Vitro Effect of Temperature on Dentin Bond Strength of Universal Adhesive Systems. Odovtos Int. J. Dent. Sci. 2020, 22, 93-101. [CrossRef]

53. Chen, Y.; Yan, X.; Li, K.; Zheng, S.; Sano, H.; Zhan, D.; Fu, J. Effect of air-blowing temperature and water storage time on the bond strength of five universal adhesive systems to dentin. Dent. Mater. J. 2020, 31, 116-122. [CrossRef]

54. Thanatvarakorn, O.; Prasansuttiporn, T.; Thittaweerat, S.; Foxton, R.M.; Ichinose, S.; Tagami, J.; Hosaka, K.; Nakajima, M. Smear layer-deproteinizing improves bonding of one-step self-etch adhesives to dentin. Dent. Mater. 2018, 34, 434-441. [CrossRef]

55. Silva, T.M.; Gonçalves, L.L.; Fonseca, B.M.; Esteves, S.R.M.S.; Barcellos, D.C.; Damião, A.J.; Gonçalves, S.E.P. Influence of Nd:YAG laser on intrapulpal temperature and bond strength of human dentin under simulated pulpal pressure. Lasers Med. Sci. 2016, 31, 49-56. [CrossRef]

56. Chasqueira, A.F.; Arantes-Oliveira, S.; Portugal, J. Bonding performance of simplified dental adhesives with three application protocols: An 18-month in vitro study. J. Adhes. Dent. 2020, 22, 255-264. [CrossRef]

57. De Lima, J.F.M.; Wajngarten, D.; Islam, F.; Clifford, J.; Botta, A.C.; De Lima, J.F.M.; Wajngarten, D.; Islam, F.; Clifford, J.; Botta, A.C. Effect of adhesive mode and chlorhexidine on microtensile strength of universal bonding agent to sound and caries-affected dentins. Eur. J. Dent. 2018, 12, 553-558. [CrossRef]

58. Flury, S.; Peutzfeldt, A.; Schmidlin, P.R.; Lussi, A. Exposed Dentin: Influence of cleaning procedures and simulated pulpal pressure on bond strength of a universal adhesive system. PLoS ONE 2017, 12, e0169680. [CrossRef] [PubMed]

59. Sutil, B.G.D.S.; Susin, A.H. Dentin pretreatment and adhesive temperature as affecting factors on bond strength of a universal adhesive system. J. Appl. Oral Sci. 2017, 25, 533-540. [CrossRef]

60. Bacelar-Sá, R.; Giannini, M.; Ambrosano, G.M.B.; Bedran-Russo, A.K. Dentin Sealing and Bond Strength Evaluation of Hema-Free and Multi-Mode Adhesives to Biomodified Dentin. Braz. Dent. J. 2017, 28, 731-737. [CrossRef]

61. Baena, E.; Cunha, S.R.; Maravić, T.; Comba, A.; Paganelli, F.; Alessandri-Bonetti, G.; Ceballos, L.; Tay, F.R.; Breschi, L.; Mazzoni, A. Effect of Chitosan as a Cross-Linker on Matrix Metalloproteinase Activity and Bond Stability with Different Adhesive Systems. Mar. Drugs 2020, 18, 263. [CrossRef] [PubMed]

62. Matinlinna, J.P.; Lung, C.Y.K.; Tsoi, J.K.H. Silane adhesion mechanism in dental applications and surface treatments: A review. Dent. Mater. 2018, 34, 13-28. [CrossRef]

63. Kaynar, Z.B.; Kazak, M.; Donmez, N.; Dalkilic, E.E. The effect of additional chlorhexidine and/or ethanol on the bond strength of universal adhesives. J. Adhes. Sci. Technol. 2021, 35, 375-385. [CrossRef]

64. Kusdemir, M.; Çetin, A.R.; Özsoy, A.; Toz, T.; Öztürk Bozkurt, F.; Özcan, M. Does 2\% chlorhexidine digluconate cavity disinfectant or sodium fluoride/hydroxyethyl methacrylate affect adhesion of universal adhesive to dentin? J. Adhes. Sci. Technol. 2016, 30, 13-23. [CrossRef]

65. Paulose, N.E.; Fawzy, A.S. Effect of grape seed extract on the bond strength and durability of resin-dentin interface. J. Adhes. Sci. Technol. 2017, 31, 2525-2541. [CrossRef]

66. Luong, M.N.; Huang, L.; Chan, D.C.N.; Sadr, A. In vitro study on the effect of a new bioactive desensitizer on dentin tubule sealing and bonding. J. Funct. Biomater. 2020, 11, 38. [CrossRef] [PubMed]

67. Siso, S.H.; Dönmez, N.; Kahya, D.S.; Uslu, Y.S. The effect of calcium phosphate-containing desensitizing agent on the microtensile bond strength of multimode adhesive agent. Niger. J. Clin. Pract. 2017, 20, 964-970. [CrossRef] 
68. Bravo, C.; Sampaio, C.S.; Hirata, R.; Puppin-Rontani, R.M.; Mayoral, J.R.; Giner, L. Effect of 2\% chlorhexidine on dentin shear bond strength of different adhesive systems: A 6 months evaluation. Int. J. Morphol. 2017, 35, 1140-1146. [CrossRef]

69. Zenobi, W.; Feitosa, V.P.; Moura, M.E.M.; D’arcangelo, C.; de Azevedo Rodrigues, L.K.; Sauro, S. The effect of zoledronatecontaining primer on dentin bonding of a universal adhesive. J. Mech. Behav. Biomed. Mater. 2018, 77, 199-204. [CrossRef]

70. Bravo, C.; Sampaio, C.S.; Hirata, R.; Puppin-Rontani, R.M.; Mayoral, J.R.; Giner, L. In-vitro Comparative Study of the use of 2\% Chlorhexidine on Microtensile Bond Strength of Different Dentin Adhesives: A 6 Months Evaluation. Int. J. Morphol. 2017, 35, 893-900. [CrossRef]

71. Ebrahimi Chaharom, M.E.; Bahari, M.; Rahbar, M.; Golbaz, S. Effect of galardin and its solvents on the microtensile bond strength of different adhesive systems to dentin. Pesqui. Bras. Odontopediatria Clin. Integr. 2019, 19, 1-12. [CrossRef]

72. Giacomini, M.C.; Scaffa, P.M.C.; Gonçalves, R.S.; Zabeu, G.S.; de Vidal, C.M.P.; de Carrilho, M.R.O.; Honório, H.M.; Wang, L. Profile of a 10-MDP-based universal adhesive system associated with chlorhexidine: Dentin bond strength and in situ zymography performance. J. Mech. Behav. Biomed. Mater. 2020, 110, 103925. [CrossRef] [PubMed]

73. Mohamed, A.M.; Nabih, S.M.; Wakwak, M.A. Effect of chitosan nanoparticles on microtensile bond strength of resin composite to dentin: An in vitro study. Brazilian Dent. Sci. 2020, 23, 1-10. [CrossRef]

74. Paulose, N.E.; Fawzy, A.S. Effect of carbodiimide on the bond strength and durability of resin-dentin interface. J. Adhes. Sci. Technol. 2018, 32, 931-946. [CrossRef]

75. Peng, W.; Yi, L.; Wang, Z.; Yang, H.; Huang, C. Effects of resveratrol/ethanol pretreatment on dentin bonding durability. Mater. Sci. Eng. C. 2020, 114, 111000. [CrossRef]

76. Shadman, N.; Farzin-Ebrahimi, S.; Mortazavi-Lahijani, E.; Jalali, Z. Effect of chlorhexidine on the durability of a new universal adhesive system. J. Clin. Exp. Dent. 2018, 10, e921-e926. [CrossRef]

77. Tekçe, N.; Tuncer, S.; Demirci, M.; Balci, S. Do matrix metalloproteinase inhibitors improve the bond durability of universal dental adhesives? Scanning 2016, 38, 535-544. [CrossRef]

78. Ahn, J.; Jung, K.-H.; Son, S.-A.; Hur, B.; Kwon, Y.-H.; Park, J.-K. Effect of additional etching and ethanol-wet bonding on the dentin bond strength of one-step self-etch adhesives. Restor. Dent. Endod. 2015, 40, 68. [CrossRef]

79. Ahmed, M.H.; De Munck, J.; Van Landuyt, K.; Peumans, M.; Yoshihara, K.; Van Meerbeek, B. Do Universal Adhesives Benefit from an Extra Bonding Layer? J. Adhes. Dent. 2019, 21, 117-132. [CrossRef] [PubMed]

80. Ermis, R.B.; Ugurlu, M.; Ahmed, M.H.; Van Meerbeek, B. Universal Adhesives Benefit from an Extra Hydrophobic Adhesive Layer When Light Cured Beforehand. J. Adhes. Dent. 2019, 21, 179-188. [CrossRef]

81. Munoz, M.A.; Sezinando, A.; Luque-Martinez, I.; Szesz, A.L.; Reis, A.; Loguercio, A.D.; Bombarda, N.H.; Perdigao, J. Influence of a hydrophobic resin coating on the bonding efficacy of three universal adhesives. J. Dent. 2014, 42, 595-602. [CrossRef] [PubMed]

82. Islam, M.S.; Hiraishi, N.; Nassar, M.; Yiu, C.; Otsuki, M.; Tagami, J.; Islama, M.S.; Hiraishi, N.; Nassar, M.; Yiu, C.; et al. Effect of hesperidin incorporation into a self-etching primer on durability of dentin bond. Dent. Mater. 2014, 30, 1205-1212. [CrossRef]

83. Perdigao, J.; Munoz, M.A.; Sezinando, A.; Luque-Martinez, I.V.; Staichak, R.; Reis, A.; Loguercio, A.D.; Perdigão, J.; Muñoz, M.A.; Sezinando, A.; et al. Immediate adhesive properties to dentin and enamel of a universal adhesive associated with a hydrophobic resin coat. Oper. Dent. 2014, 39, 489-499. [CrossRef]

84. Sezinando, A.; Luque-Martinez, I.; Muñoz, M.A.; Reis, A.; Loguercio, A.D.; Perdigão, J.; Munoz, M.A.; Reis, A.; Loguercio, A.D.; Perdigao, J.; et al. Influence of a hydrophobic resin coating on the immediate and 6-month dentin bonding of three universal adhesives. Dent. Mater. 2015, 31, e236-e246. [CrossRef] [PubMed]

85. Vinagre, A.; Ralho, A.; Ramos, N.; Messias, A.; Ramos, J. Bonding performance of a universal adhesive: Effect of hydrophobic resin coating and long-term water storage. Rev. Port. Estomatol. Med. Dentária Cir. Maxilofac. 2019, 60. [CrossRef]

86. Cadenaro, M.; Maravic, T.; Comba, A.; Mazzoni, A.; Fanfoni, L.; Hilton, T.; Ferracane, J.; Breschi, L. The role of polymerization in adhesive dentistry. Dent. Mater. 2019, 35, e1-e22. [CrossRef]

87. Benitez Sellan, P.L.; Zanatta, R.F.; Gomes Torres, C.R.; Tian, F.; Bergeron, B.E.; Niu, L.; Pucci, C.R. Effects of calcium-phosphate, laser and adhesive on dentin permeability and bond strength. Heliyon 2020, 6, e03925. [CrossRef]

88. Shadman, N.; Farzin Ebrahimi, S.; Amanpour, S.; Mehdizadeh, S. Shear Bond Strength of a Multi-Mode Adhesive to Bur-Cut and Er,Cr:YSGG Lased Dentin in Different Output Powers. J. Dent. 2019, 20, 118-123. [CrossRef]

89. Silva, A.C.; Melo, P.; Ferreira, J.; Oliveira, S.; Gutknecht, N. Influence of grape seed extract in adhesion on dentin surfaces conditioned with Er,Cr:YSGG laser. Lasers Med. Sci. 2019, 34, 1493-1501. [CrossRef]

90. Rechmann, P.; Bartolome, N.; Kinsel, R.; Vaderhobli, R.; Rechmann, B.M.T. Bond strength of etch-and-rinse and self-etch adhesive systems to enamel and dentin irradiated with a novel CO2 $9.3 \mu \mathrm{m}$ short-pulsed laser for dental restorative procedures. Lasers Med. Sci. 2017, 32, 1981-1993. [CrossRef]

91. Trevelin, L.T.; da Silva, B.T.F.; de Freitas, P.M.; Matos, A.B. Influence of Er:YAG laser pulse duration on the long-term stability of organic matrix and resin-dentin interface. Lasers Med. Sci. 2019, 34, 1391-1399. [CrossRef] [PubMed]

92. Yazici, A.R.; Karaman, E.; Tuncer, D.; Berk, G.; Ertan, A. Effect of an Er, Cr: YSGG laser preparation on dentin bond strength of a universal adhesive. J. Adhes. Sci. Technol. 2016, 30, 2477-2484. [CrossRef]

93. Chowdhury, A.F.M.A.; Saikaew, P.; Alam, A.; Sun, J.; Carvalho, R.M.; Sano, H. Effects of Double Application of Contemporary Self-Etch Adhesives on Their Bonding Performance to Dentin with Clinically Relevant Smear Layers. J. Adhes. Dent. 2019, 21, 59-66. [CrossRef] [PubMed] 
94. Chowdhury, A.F.M.A.; Islam, R.; Alam, A.; Matsumoto, M.; Yamauti, M.; Carvalho, R.M.; Sano, H. Variable smear layer and adhesive application: The pursuit of clinical relevance in bond strength testing. Int. J. Mol. Sci. 2019, 20, 5381. [CrossRef]

95. Fujiwara, S.; Takamizawa, T.; Barkmeier, W.W.; Tsujimoto, A.; Imai, A.; Watanabe, H.; Erickson, R.L.; Latta, M.A.; Nakatsuka, T.; Miyazaki, M. Effect of double-layer application on bond quality of adhesive systems. J. Mech. Behav. Biomed. Mater. 2018, 77, 501-509. [CrossRef]

96. Pashaev, D.; Demirci, M.; Tekce, N.; Tuncer, S.; Baydemir, C. The effect of double-coating and times on the immediate and 6-month dentin bonding of universal adhesives. Biomed. Mater. Eng. 2017, 28, 169-185. [CrossRef]

97. Taschner, M.; Kümmerling, M.; Lohbauer, U.; Breschi, L.; Petschelt, A.; Frankenberger, R.; Kummerling, M.; Lohbauer, U.; Breschi, L.; Petschelt, A.; et al. Effect of double-layer application on dentin bond durability of onestep self-etch adhesives. Oper. Dent. 2014, 39, 416-426. [CrossRef] [PubMed]

98. Zecin-Deren, A.; Sokolowski, J.; Szczesio-Wlodarczyk, A.; Piwonski, I.; Lukomska-Szymanska, M.; Lapinska, B. Multi-layer application of self-etch and universal adhesives and the effect on dentin bond strength. Molecules 2019, 24, 345. [CrossRef] [PubMed]

99. Zecin-Deren, A.; Lukomska-Szymanska, M.; Szczesio-Wlodarczyk, A.; Piwonski, I.; Sokolowski, J.; Lapinska, B. The influence of application protocol of simplified and universal adhesives on the dentin bonding performance. Appl. Sci. 2020, 10, 124. [CrossRef]

100. Ayres, A.P.; Bonvent, J.J.; Mogilevych, B.; Soares, L.E.S.; Martin, A.A.; Ambrosano, G.M.; Nascimento, F.D.; Van Meerbeek, B.; Giannini, M. Effect of non-thermal atmospheric plasma on the dentin-surface topography and composition and on the bond strength of a universal adhesive. Eur. J. Oral Sci. 2018, 126, 53-65. [CrossRef]

101. Ayres, A.; Freitas, P.; De Munck, J.; Vananroye, A.; Clasen, C.; Dias, C.D.S.; Giannini, M.; Van Meerbeek, B. Benefits of Nonthermal Atmospheric Plasma Treatment on Dentin Adhesion. Oper. Dent. 2018, 43, E288-E299. [CrossRef] [PubMed]

102. Alqahtani, M.Q. Influence of acid-etching or double-curing time on dentin bond strength of one-step self-etch adhesive. Saudi J. Dent. Res. 2015, 6, 110-116. [CrossRef]

103. Sampaio, P.C.P.; de Kruly, P.C.; Ribeiro, C.C.; Hilgert, L.A.; Pereira, P.N.R.; Scaffa, P.M.C.; Di Hipólito, V.; D’Alpino, P.H.P.; Garcia, F.C.P. Comparative bonding ability to dentin of a universal adhesive system and monomer conversion as functions of extended light curing times and storage. J. Mech. Behav. Biomed. Mater. 2017, 75, 41-49. [CrossRef] [PubMed]

104. Fu, J.; Saikaew, P.; Kawano, S.; Carvalho, R.M.; Hannig, M.; Sano, H.; Selimovic, D. Effect of air-blowing duration on the bond strength of current one-step adhesives to dentin. Dent. Mater. 2017, 33, 895-903. [CrossRef] [PubMed]

105. Luque-Martinez, I.V.; Perdigão, J.; Muñoz, M.A.; Sezinando, A.; Reis, A.; Loguercio, A.D. Effects of solvent evaporation time on immediate adhesive properties of universal adhesives to dentin. Dent. Mater. 2014, 30, 1126-1135. [CrossRef] [PubMed]

106. Saikaew, P.; Matsumoto, M.; Chowdhury, A.; Carvalho, R.; Sano, H. Does Shortened Application Time Affect Long-Term Bond Strength of Universal Adhesives to Dentin? Oper. Dent. 2018, 43, 549-558. [CrossRef] [PubMed]

107. Irmak, Ö.; Yaman, B.C.; Orhan, E.O.; Ozer, F.; Blatz, M.B. Effect of rubbing force magnitude on bond strength of universal adhesives applied in self-etch mode. Dent. Mater. J. 2018, 37, 139-145. [CrossRef]

108. Jang, J.H.; Jeon, B.K.; Mo, S.Y.; Park, M.; Choi, D.; Choi, K.K.; Kim, D.S. Effect of various agitation methods on adhesive layer formation of hema-free universal dentin adhesive. Dent. Mater. J. 2019, 38, 101-106. [CrossRef]

109. Thanatvarakorn, O.; Prasansuttiporn, T.; Takahashi, M.; Thittaweerat, S.; Foxton, R.M.; Ichinose, S.; Tagami, J.; Nakajima, M. Effect of Scrubbing Technique with Mild Self-etching Adhesives on Dentin Bond Strengths and Nanoleakage Expression. J. Adhes. Dent. 2016, 18, 197-204. [CrossRef]

110. Kharouf, N.; Rapp, G.; Mancino, D.; Hemmerlé, J.; Haikel, Y.; Reitzer, F. Effect of etching the coronal dentin with the rubbing technique on the microtensile bond strength of a universal adhesive system. Dent. Med. Probl. 2019, 56, 343-348. [CrossRef]

111. Stape, T.H.S.; Wik, P.; Mutluay, M.M.; Al-Ani, A.A.S.; Tezvergil-Mutluay, A. Selective dentin etching: A potential method to improve bonding effectiveness of universal adhesives. J. Mech. Behav. Biomed. Mater. 2018, 86, 14-22. [CrossRef]

112. Takamizawa, T.; Barkmeier, W.W.; Tsujimoto, A.; Suzuki, T.; Scheidel, D.D.; Erickson, R.L.; Latta, M.A.; Miyazaki, M. Influence of different pre-etching times on fatigue strength of self-etch adhesives to dentin. Eur. J. Oral Sci. 2016. [CrossRef] [PubMed]

113. Saikaew, P.; Fu, J.; Chowdhury, A.F.M.A.M.A.; Carvalho, R.M.; Sano, H. Effect of air-blowing time and long-term storage on bond strength of universal adhesives to dentin. Clin. Oral Investig. 2019, 23, 2629-2635. [CrossRef]

114. Amsler, F.; Peutzfeldt, A.; Lussi, A.; Flury, S. Bond strength of resin composite to dentin with different adhesive systems: Influence of relative humidity and application time. J. Adhes. Dent. 2015, 17, 249-256. [CrossRef] [PubMed]

115. Huang, X.Q.; Pucci, C.R.; Luo, T.; Breschi, L.; Pashley, D.H.; Niu, L.N.; Tay, F.R. No-waiting dentine self-etch concept-Merit or hype. J. Dent. 2017. [CrossRef] [PubMed]

116. Saikaew, P.; Chowdhury, A.F.M.A.; Fukuyama, M.; Kakuda, S.; Carvalho, R.M.; Sano, H. The effect of dentine surface preparation and reduced application time of adhesive on bonding strength. J. Dent. 2016, 47, 63-70. [CrossRef]

117. Peumans, M.; Kanumilli, P.; De Munck, J.; Van Landuyt, K.; Lambrechts, P.; Van Meerbeek, B. Clinical effectiveness of contemporary adhesives: A systematic review of current clinical trials. Dent. Mater. 2005, 21, 864-881. [CrossRef]

118. Nunes, T.G.; Ceballos, L.; Osorio, R.; Toledano, M. Spatially resolved photopolymerization kinetics and oxygen inhibition in dental adhesives. Biomaterials 2005, 26, 1809-1817. [CrossRef]

119. Perdigao, J.; Lambrechts, P.; Van Meerbeek, B.; Braem, M.; Yildiz, E.; Yucel, T.; Vanherle, G. The interaction of adhesive systems with human dentin. Am. J. Dent. 1996, 9, 167-173. 
120. Albuquerque, M.; Pegoraro, M.; Mattei, G.; Reis, A.; Loguercio, A.D. Effect of Double-application or the Application of a Hydrophobic Layer for Improved Efficacy of One-step Self-etch Systems in Enamel and Dentin. Oper. Dent. 2008, 33, 564-570. [CrossRef] [PubMed]

121. Reis, A.; Albuquerque, M.; Pegoraro, M.; Mattei, G.; de Bauer, J.R.O.; Grande, R.H.M.; Klein-Junior, C.A.; Baumhardt-Neto, R.; Loguercio, A.D. Can the durability of one-step self-etch adhesives be improved by double application or by an extra layer of hydrophobic resin? J. Dent. 2008, 36, 309-315. [CrossRef]

122. Sartori, N.; Peruchi, L.D.; Guimarães, J.C.; Silva, S.B.; Monteiro Jr, S.; Baratieri, L.N.; Belli, R. Clinical effectiveness of a hydrophobic coating used in conjunction with a one-step self-etch adhesive: An 18-month evaluation. Oper. Dent. 2013, 38, 249-257. [CrossRef] [PubMed]

123. Breschi, L.; Mazzoni, A.; Ruggeri, A.; Cadenaro, M.; Di Lenarda, R.; De Stefano Dorigo, E. Dental adhesion review: Aging and stability of the bonded interface. Dent. Mater. 2008, 24, 90-101. [CrossRef] [PubMed]

124. Loguercio, A.D.; Loeblein, F.; Cherobin, T.; Ogliari, F.; Piva, E.; Reis, A. Effect of solvent removal on adhesive properties of simplified etch-and-rinse systems and on bond strengths to dry and wet dentin. J. Adhes. Dent. 2009, 11, 213-219. [CrossRef]

125. Cadenaro, M.; Antoniolli, F.; Sauro, S.; Tay, F.R.; Di Lenarda, R.; Prati, C.; Biasotto, M.; Contardo, L.; Breschi, L. Degree of conversion and permeability of dental adhesives. Eur. J. Oral Sci. 2005, 113, 525-530. [CrossRef]

126. Cadenaro, M.; Navarra, C.O.; Antoniolli, F.; Mazzoni, A.; Di Lenarda, R.; Rueggeberg, F.A.; Breschi, L. The effect of curing mode on extent of polymerization and microhardness of dual-cured, self-adhesive resin cements. Am. J. Dent. 2010, $23,14-18$.

127. Breschi, L.; Cadenaro, M.; Antoniolli, F.; Sauro, S.; Biasotto, M.; Prati, C.; Tay, F.R.; Di Lenarda, R. Polymerization kinetics of dental adhesives cured with LED: Correlation between extent of conversion and permeability. Dent. Mater. 2007, 23, 1066-1072. [CrossRef] [PubMed]

128. Ito, S.; Tay, F.R.; Hashimoto, M.; Yoshiyama, M.; Saito, T.; Brackett, W.W.; Waller, J.L.; Pashley, D.H. Effects of multiple coatings of two all-in-one adhesives on dentin bonding. J. Adhes. Dent. 2005, 7, 133-141. [PubMed]

129. Yoshihara, K.; Yoshida, Y.; Hayakawa, S.; Nagaoka, N.; Irie, M.; Ogawa, T.; Van Landuyt, K.L.; Osaka, A.; Suzuki, K.; Minagi, S.; et al. Nanolayering of phosphoric acid ester monomer on enamel and dentin. Acta Biomater. 2011, 7, $3187-3195$. [CrossRef]

130. Yiu, C.K.Y.; Pashley, E.L.; Hiraishi, N.; King, N.M.; Goracci, C.; Ferrari, M.; Carvalho, R.M.; Pashley, D.H.; Tay, F.R. Solvent and water retention in dental adhesive blends after evaporation. Biomaterials 2005, 26, 6863-6872. [CrossRef]

131. Bedran-Russo, A.; Leme-Kraus, A.A.; Vidal, C.M.P.; Teixeira, E.C. An Overview of Dental Adhesive Systems and the Dynamic Tooth-Adhesive Interface. Dent. Clin. North Am. 2017, 61, 713-731. [CrossRef]

132. Seara, S.F.; Erthal, B.S.; Ribeiro, M.; Kroll, L.; Pereira, G.D.S. The influence of a dentin desensitizer on the microtensile bond strength of two bonding systems. Oper. Dent. 2002, 27, 154-160. [PubMed]

133. Sengun, A.; Koyuturk, A.E.; Sener, Y.; Ozer, F. Effect of desensitizers on the bond strength of a self-etching adhesive system to caries-affected dentin on the gingival wall. Oper. Dent. 2005, 30, 430-435.

134. Yang, H.; Pei, D.; Chen, Z.; Lei, J.; Zhou, L.; Huang, C. Effects of the application sequence of calcium-containing desensitising pastes during etch-and-rinse adhesive restoration. J. Dent. 2014. [CrossRef] [PubMed]

135. Sahrmann, P.; Ronay, V.; Schmidlin, P.R.; Attin, T.; Paqué, F. Three-Dimensional Defect Evaluation of Air Polishing on Extracted Human Roots. J. Periodontol. 2014. [CrossRef] [PubMed]

136. Tay, F.R.; Pashley, D.H.; Kapur, R.R.; Carrilho, M.R.O.; Hur, Y.B.; Garrett, L.V.; Tay, K.C.Y. Bonding BisGMA to Dentin-a Proof of Concept for Hydrophobic Dentin Bonding. J. Dent. Res. 2007, 86, 1034-1039. [CrossRef]

137. Shin, T.P.; Yao, X.; Huenergardt, R.; Walker, M.P.; Wang, Y. Morphological and chemical characterization of bonding hydrophobic adhesive to dentin using ethanol wet bonding technique. Dent. Mater. 2009, 25, 1050-1057. [CrossRef]

138. Sauro, S.; Toledano, M.; Aguilera, F.S.; Mannocci, F.; Pashley, D.H.; Tay, F.R.; Watson, T.F.; Osorio, R. Resin-dentin bonds to EDTA-treated vs. acid-etched dentin using ethanol wet-bonding. Part II: Effects of mechanical cycling load on microtensile bond strengths. Dent. Mater. 2011, 27, 563-572. [CrossRef]

139. Liu, Y.; Liu, Q.; Yu, Q.S.; Wang, Y. Nonthermal Atmospheric Plasmas in Dental Restoration. J. Dent. Res. 2016, 95, 496-505. [CrossRef]

140. Han, G.J.; Kim, J.H.; Chung, S.N.; Chun, B.H.; Kim, C.K.; Seo, D.G.; Son, H.H.; Cho, B.H. Effects of non-thermal atmospheric pressure pulsed plasma on the adhesion and durability of resin composite to dentin. Eur. J. Oral Sci. 2014. [CrossRef]

141. Chen, M.; Zhang, Y.; Sky Driver, M.; Caruso, A.N.; Yu, Q.; Wang, Y. Surface modification of several dental substrates by non-thermal, atmospheric plasma brush. Dent. Mater. 2013, 29, 871-880. [CrossRef]

142. Zhang, Y.; Yu, Q.; Wang, Y. Non-thermal atmospheric plasmas in dental restoration: Improved resin adhesive penetration. J. Dent. 2014, 42, 1033-1042. [CrossRef]

143. Ritts, A.C.; Li, H.; Yu, Q.; Xu, C.; Yao, X.; Hong, L.; Wang, Y. Dentin surface treatment using a non-thermal argon plasma brush for interfacial bonding improvement in composite restoration. Eur. J. Oral Sci. 2010. [CrossRef] [PubMed]

144. Kim, J.-H.; Han, G.-J.; Kim, C.-K.; Oh, K.-H.; Chung, S.-N.; Chun, B.-H.; Cho, B.-H. Promotion of adhesive penetration and resin bond strength to dentin using non-thermal atmospheric pressure plasma. Eur. J. Oral Sci. 2016, 124, 89-95. [CrossRef]

145. Chen, M.; Zhang, Y.; Dusevich, V.; Liu, Y.; Yu, Q.; Wang, Y. Non-thermal atmospheric plasma brush induces HEMA grafting onto dentin collagen. Dent. Mater. 2014, 30, 1369-1377. [CrossRef] [PubMed] 
146. Van Meerbeek, B.; De Munck, J.; Yoshida, Y.; Inoue, S.; Vargas, M.; Vijay, P.; Van Landuyt, K.; Lambrechts, P.; Vanherle, G. Buonocore memorial lecture. Adhesion to enamel and dentin: Current status and future challenges. Oper. Dent. 2003, 28, 215-235.

147. Wagner, A.; Wendler, M.; Petschelt, A.; Belli, R.; Lohbauer, U. Bonding performance of universal adhesives in different etching modes. J. Dent. 2014, 42, 800-807. [CrossRef]

148. Reis, A.; Carrilho, M.; Breschi, L.; Loguercio, A.D. Overview of clinical alternatives to minimize the degradation of the resin-dentin bonds. Oper. Dent. 2013, 38, E103-E127. [CrossRef] [PubMed]

149. Reis, A.; Pellizzaro, A.; Dal-Bianco, K.; Gomes, O.M.; Patzlaff, R.; Loguercio, A.D. Impact of Adhesive Application to Wet and Dry Dentin on Long-term Resin-dentin Bond Strengths. Oper. Dent. 2007, 32, 380-387. [CrossRef] [PubMed]

150. Yoshida, Y.; Yoshihara, K.; Nagaoka, N.; Hayakawa, S.; Torii, Y.; Ogawa, T.; Osaka, A.; Meerbeek, B. Van Self-assembled nano-layering at the adhesive interface. J. Dent. Res. 2012, 91, 376-381. [CrossRef] [PubMed]

151. Inoue, S.; Vargas, M.A.; Abe, Y.; Yoshida, Y.; Lambrechts, P.; Vanherle, G.; Sano, H.; Van Meerbeek, B. Microtensile bond strength of eleven contemporary adhesives to dentin. J. Adhes. Dent. 2001, 3, 237-245. [PubMed]

152. Cardoso, M.V.; Delmé, K.I.M.; Mine, A.; Neves, A.D.A.; Coutinho, E.; De Moor, R.J.G.; Van Meerbeek, B. Towards a better understanding of the adhesion mechanism of resin-modified glass-ionomers by bonding to differently prepared dentin. J. Dent. 2010, 38, 921-929. [CrossRef]

153. De Munck, J.; Van Meerbeek, B.; Yudhira, R.; Lambrechts, P.; Vanherle, G. Micro-tensile bond strength of two adhesives to Erbium:YAG-lased vs. bur-cut enamel and dentin. Eur. J. Oral Sci. 2002, 110, 322-329. [CrossRef]

154. Aoki, A.; Ishikawa, I.; Yamada, T.; Otsuki, M.; Watanabe, H.; Tagami, J.; Ando, Y.; Yamamoto, H. Comparison between Er:YAG laser and conventional technique for root caries treatment in vitro. J. Dent. Res. 1998. [CrossRef]

155. Li, Z.-Z.; Code, J.E.; Van de Merwe, W.P. Er:YAG laser ablation of enamel and dentin of human teeth: Determination of ablation rates at various fluences and pulse repetition rates. Lasers Surg. Med. 1992, 12, 625-630. [CrossRef]

156. Comba, A.; Baldi, A.; Michelotto Tempesta, R.; Cedrone, A.; Carpegna, G.; Mazzoni, A.; Breschi, L.; Alovisi, M.; Pasqualini, D.; Scotti, N.; et al. Effect of Er:YAG and Burs on Coronal Dentin Bond Strength Stability. J. Adhes. Dent. 2019, 21, 329-335. [CrossRef]

157. Samad-Zadeh, A.; Harsono, M.; Belikov, A.; Shatilova, K.V.; Skripnik, A.; Stark, P.; Egles, C.; Kugel, G. The influence of laser-textured dentinal surface on bond strength. Dent. Mater. 2011, 27, 1038-1044. [CrossRef]

158. Ikeda, T.; De Munck, J.; Shirai, K.; Hikita, K.; Inoue, S.; Sano, H.; Lambrechts, P.; Van Meerbeek, B. Effect of evaporation of primer components on ultimate tensile strengths of primer-adhesive mixture. Dent. Mater. 2005, 21, 1051-1058. [CrossRef]

159. Mine, A.; De Munck, J.; Cardoso, M.V.; Van Landuyt, K.L.; Poitevin, A.; Van Ende, A.; Matsumoto, M.; Yoshida, Y.; Kuboki, T.; Yatani, H.; et al. Dentin-smear remains at self-etch adhesive interface. Dent. Mater. 2014, 30, 1147-1153. [CrossRef]

160. Carvalho, R.M.; Mendonça, J.S.; Santiago, S.L.; Silveira, R.R.; Garcia, F.C.P.; Tay, F.R.; Pashley, D.H. Effects of HEMA/solvent combinations on bond strength to dentin. J. Dent. Res. 2003, 82, 597-601. [CrossRef]

161. Pashley, D.H.; Ciucchi, B.; Sano, H.; Horner, J.A. Permeability of dentin to adhesive agents. Quintessence Int. 1993, $24,618-631$.

162. Reis, A.; de Carvalho Cardoso, P.; Vieira, L.C.C.; Baratieri, L.N.; Grande, R.H.M.; Loguercio, A.D. Effect of prolonged application times on the durability of resin-dentin bonds. Dent. Mater. 2008, 24, 639-644. [CrossRef]

163. Yoshihara, K.; Yoshida, Y.; Hayakawa, S.; Nagaoka, N.; Torii, Y.; Osaka, A.; Suzuki, K.; Minagi, S.; Van Meerbeek, B.; Van Landuyt, K.L. Self-etch Monomer-Calcium Salt Deposition on Dentin. J. Dent. Res. 2011, 90, 602-606. [CrossRef]

164. Manuja, N.; Nagpal, R.; Pandit, I.K. Dental adhesion: Mechanism, techniques and durability. J. Clin. Pediatr. Dent. 2012, 36, $223-234$. [CrossRef]

165. Hass, V.; Luque-Martinez, I.V.; Gutierrez, M.F.; Moreira, C.G.; Gotti, V.B.; Feitosa, V.P.; Koller, G.; Otuki, M.F.; Loguercio, A.D.; Reis, A. Collagen cross-linkers on dentin bonding: Stability of the adhesive interfaces, degree of conversion of the adhesive, cytotoxicity and in situ MMP inhibition. Dent. Mater. 2016, 32, 732-741. [CrossRef]

166. Daood, U.; Yiu, C.K.Y.; Burrow, M.F.; Niu, L.-N.; Tay, F.R. Effect of a novel quaternary ammonium silane on dentin protease activities. J. Dent. 2017, 58, 19-27. [CrossRef]

167. Favetti, M.; Schroeder, T.; Montagner, A.F.; Correa, M.B.; Pereira-Cenci, T.; Cenci, M.S. Effectiveness of pre-treatment with chlorhexidine in restoration retention: A 36-month follow-up randomized clinical trial. J. Dent. 2017, 60, 44-49. [CrossRef]

168. Mazzoni, A.; Angeloni, V.; Apolonio, F.M.; Scotti, N.; Tjäderhane, L.; Tezvergil-Mutluay, A.; Di Lenarda, R.; Tay, F.R.; Pashley, D.H.; Breschi, L. Effect of carbodiimide (EDC) on the bond stability of etch-and-rinse adhesive systems. Dent. Mater. 2013, 29, 1040-1047. [CrossRef]

169. Carvalho, C.; Fernandes, F.P.; Freitas, V.D.P.; França, F.M.G.; Basting, R.T.; Turssi, C.P.; Amaral, F.L.B. Effect of green tea extract on bonding durability of an etch-and-rinse adhesive system to caries-affected dentin. J. Appl. Oral Sci. 2016, 24, 211-217. [CrossRef]

170. de Carvalho, R.V.; Vieira, E.O.; Gaviolli, E.; Graunke, A.; Chisini, L.A.; Bacchi, A.; Ghiggi, P.C.; van de Sande, F.H. Doxycycline as a dentin pretreatment agent for MMP-2 inhibition and maintaining hybrid layer stability over time. Int. J. Adhes. Adhes. 2020, 98, 102510. [CrossRef]

171. Münchow, E.A.; Bottino, M.C. Recent Advances in Adhesive Bonding: The Role of Biomolecules, Nanocompounds, and Bonding Strategies in Enhancing Resin Bonding to Dental Substrates. Curr. Oral Heal. Reports 2017, 4, 215-227. [CrossRef]

172. Bailey, A.J.; Light, N.D.; Atkins, E.D.T. Chemical cross-linking restrictions on models for the molecular organization of the collagen fibre. Nature 1980, 288, 408-410. [CrossRef]

173. Fawzy, A.S.; Nitisusanta, L.I.; Iqbal, K.; Daood, U.; Neo, J. Riboflavin as a dentin crosslinking agent: Ultraviolet A versus blue light. Dent. Mater. 2012, 28, 1284-1291. [CrossRef] 
174. Bourgi, R.; Daood, U.; Bijle, M.N.; Fawzy, A.; Ghaleb, M.; Hardan, L. Reinforced Universal Adhesive by Ribose Crosslinker: A Novel Strategy in Adhesive Dentistry. Polymers 2021, 13, 704. [CrossRef]

175. Kishen, A.; Shrestha, S.; Shrestha, A.; Cheng, C.; Goh, C. Characterizing the collagen stabilizing effect of crosslinked chitosan nanoparticles against collagenase degradation. Dent. Mater. 2016, 32, 968-977. [CrossRef]

176. Daood, U.; Sauro, S.; Pichika, M.R.; Omar, H.; Liang Lin, S.; Fawzy, A.S. Novel riboflavin/VE-TPGS modified universal dentine adhesive with superior dentine bond strength and self-crosslinking potential. Dent. Mater. 2020, 36, 145-156. [CrossRef]

177. Faggion, C.M., Jr. Guidelines for reporting pre-clinical in vitro studies on dental materials. J. Evid. Based Dent. Pract. 2012, 12, 182-189. [CrossRef] 